Preprint typeset using $\mathrm{AT}_{\mathrm{E}} \mathrm{X}$ style emulateapj v. 5/2/11

\title{
THE EMBEDDED RING-LIKE FEATURE AND STAR FORMATION ACTIVITIES IN G35.673-00.847
}

\author{
L. K. Dewangan ${ }^{1}$, R. Devaraj ${ }^{2}$, and D. K. Ojha ${ }^{3}$
}

\begin{abstract}
We present a multi-wavelength study to probe the star formation (SF) process in the molecular cloud linked with the G35.673-00.847 site (hereafter MCG35.6), which is traced in a velocity range of 53-62 $\mathrm{km} \mathrm{s}^{-1}$. Multi-wavelength images reveal a semi-ring-like feature (associated with ionized gas emission) and an embedded face-on ring-like feature (without the NVSS $1.4 \mathrm{GHz}$ radio emission; where $1 \sigma \sim 0.45$ mJy beam $^{-1}$ ) in the MCG35.6. The semi-ring-like feature is originated by the ionizing feedback from a star with spectral type B0.5V-B0V. The central region of the ring-like feature does not contain detectable ionized gas emission, indicating that the ring-like feature is unlikely to be produced by the ionizing feedback from a massive star. Several embedded Herschel clumps and young stellar objects (YSOs) are identified in the MCG35.6, tracing the ongoing SF activities within the cloud. The polarization information from the Planck and GPIPS data trace the plane-of-sky magnetic field, which is oriented parallel to the major axis of the ring-like feature. At least five clumps (having $M_{\text {clump }} \sim 740-1420 \mathrm{M}_{\odot}$ ) seem to be distributed in an almost regularly spaced manner along the ringlike feature and contain noticeable YSOs. Based on the analysis of the polarization and molecular line data, three subregions containing the clumps are found to be magnetically supercritical in the ring-like feature. Altogether, the existence of the ring-like feature and the SF activities on its edges can be explained by the magnetic field mediated process as simulated by Li \& Nakamura (2002).

Subject headings: dust, extinction - HII regions - ISM: clouds - ISM: individual objects (G35.67300.847) - ISM: Magnetic fields - stars: formation
\end{abstract}

\section{INTRODUCTION}

In recent years, space-based infrared observations have demonstrated that the infrared structures (such as, bubbles, ring-like features, and shells) are often associated with young stellar clusters, and are commonly observed in Galactic molecular clouds (e.g. Churchwell et al. 2006 2007; Anderson et al. 2009; Beaumont \& Williams 2010, Deharveng et al. 2010). Churchwell et al. (2006) reported that $25 \%$ of 322 mid-infrared (MIR) bubbles, distributed outside $10^{\circ}$ of the Galactic center, are associated with known $\mathrm{H}$ II regions detected at radio wavelengths. Furthermore, using the same catalog given in Churchwell et al. (2006), Deharveng et al. (2010) extended this information to $86 \%$ out of 102 bubbles through the detection of the $20 \mathrm{~cm}$ radio continuum emission. Hence, based on the detection/non-detection of the centimeter radio continuum emission at the sensitivity of the observed radio map, the infrared structures are seen either associated with $\mathrm{H}$ II regions or devoid of $\mathrm{H}$ II regions. The infrared structures associated with $\mathrm{H}$ II regions are promising sites to investigate the feedback processes of massive OB stars (e.g. Deharveng et al. 2010, Kendrew et al. 2012, Thompson et al. 2012, Dewangan et al. 2016 2017a). The photoionized gas and/or stellar winds associated with the OB stars are often considered as the major contributor for the origin of the infrared structures. Another possible mechanism responsible for the infrared

${ }^{2}$ Instituto Nacional de Astrofísica, Óptica y Electrónica, Luis Enrique Erro \# 1, Tonantzintla, Puebla, México C.P. 72840.

${ }^{3}$ Department of Astronomy and Astrophysics, Tata Institute of Fundamental Research, Homi Bhabha Road, Mumbai 400 005, India.
}

structures could be supernovae explosions. On the other hand, the existence of a ring-like morphology without $\mathrm{H}$ II regions is unlikely to be explained by the feedback of OB stars. The magnetic field mediated scenario has predicted the formation of ring-like features in magnetically subcritical clouds (e.g. Nakamura \& Li (2002); Li \& Nakamura (2002) and references therein). These authors also examined the evolution of these subcritical clouds/ring-like features, and found that a subcritical cloud fragments into multiple magnetically supercritical cores/clumps, where the birth of young stellar populations is expected. Recently, Pavel \& Clemens (2012) examined the interaction of $\mathrm{H}$ II region driven Galactic bubbles with the Galactic magnetic field, and found that external magnetic fields are important during the earliest phases of the evolution of $\mathrm{H}$ II region driven Galactic bubbles. However, to our knowledge, a detailed observational study of the influence of the magnetic field on bubble/ring-like structures is still limited in the literature (e.g., Chen et al. 2017). Interestingly, the presence of infrared structures with $\mathrm{H}$ II regions and without $\mathrm{H}$ II regions in a single star-forming site can offer the possibility to explore the numerous complex physical processes involved in star formation. A detailed multi-wavelength study of such site can provide important observational evidences which can directly lead to examine the existing theoretical scenarios concerning the formation of stellar clusters and ring-like features not associated with $\mathrm{H}$ II regions.

The IRAS 18569+0159/G35.673-00.847 region (hereafter G35.6) is located at a distance of $3.7 \mathrm{kpc}$ (Paron et al. 2011) and is a poorly explored star-forming site. Using the radio continuum $1.4 \mathrm{GHz}$ map from the NRAO VLA Sky Survey (NVSS; 1.4 GHz; Condon et al. 1998), Paron et al. (2011) studied the $\mathrm{H}$ II region associated with 
the G35.6 site (hereafter G35.6 H II region; see Figure 1 in this paper). Based on the MIR and radio continuum emission, the G35.6 site has an almost semi-ring-like appearance with a radius of about $1.5(\sim 1.6 \mathrm{pc}$ at a distance of $3.7 \mathrm{kpc}$ ) (Paron et al. 2011), which is associated with the main radio continuum source detected in the region (see Figures 1 $\mathrm{a}$ and 1b). In the direction of the G35.6 $\mathrm{H}$ II region, the velocity of the ionized gas is estimated to be $60.7 \pm 2.6 \mathrm{~km} \mathrm{~s}^{-1}$ (Lockman|1989). Using the Galactic Ring Survey (GRS; Jackson et al. 2006$){ }^{13} \mathrm{CO}(\mathrm{J}=1-0)$ line data, Anderson et al. (2009) computed the radial velocity of the molecular gas to be $\sim 57.1 \mathrm{~km} \mathrm{~s}^{-1}$ and designated the molecular cloud associated with the G35.6 site (hereafter MCG35.6) as D35.67-0.85. Later, using the GRS ${ }^{13} \mathrm{CO}$ line data, Paron et al. (2011) reported the presence of a molecular shell (with a mass $M_{\text {cloud }} \sim 10^{4}$ $\mathrm{M}_{\odot}$ ) containing several young stellar objects (YSOs, see Figure 1 $1 \mathrm{~b}$ ). The YSOs were identified using the Two Micron AII Sky Survey (2MASS; $\lambda=1.25,1.65,2.2 \mu \mathrm{m}$; Skrutskie et al. 2006) and the Galactic Legacy Infrared Mid-Plane Survey Extraordinaire (GLIMPSE; $\lambda=3.6$, 4.5, 5.8, $8.0 \mu \mathrm{m}$; Benjamin et al. 2003) photometric data sets. the majority of the ${ }^{13} \mathrm{CO}$ gas in the molecular shell is found away from the radio continuum emission (see Figure 4 in Paron et al. 2011). In this paper, Figures 1 a and $1 \mathrm{~b}$ show the observed ionized and molecular features previously reported by Paron et al. (2011), and also reveal that the G35.6 H II region is spatially seen in the Galactic northern side of the molecular shell. Paron et al. (2011) suggested that the G35.6 H II region is interacting with its surrounding molecular cloud. However, the origin of the infrared and molecular structures seen in the G35.6 site still remains unexplored. The physical conditions in the G35.6 site are yet to be probed. Furthermore, the magnetic field properties in the molecular structure have not yet been studied. The investigation of dust continuum clumps and embedded young stellar clusters in the MCG35.6 is also yet to be carried out.

In this paper, to understand the ongoing physical processes and star formation activity in the G35.6 site, we present a detailed multi-wavelength study of observations from near-infrared (NIR) to radio wavelengths. The multi-wavelength data sets (including imaging, spectroscopic, and polarimetric observations) are obtained from various Galactic plane surveys. A careful investigation of these data sets allows us to probe the distribution of column density, extinction, dust temperature, ionized gas emission, kinematics of ${ }^{13} \mathrm{CO}$ gas, plane-of-sky (POS) magnetic field, and YSOs in the MCG35.6.

This paper is structured as follows. In Section 2, we discuss the data selection. The results of our extensive multi-wavelength data analysis are presented in Section 3 The implications of our findings concerning the star formation are discussed in Section 4. Finally, our results are summarized and concluded in Section 5 .

\section{DATA SETS AND ANALYSIS}

In this section, we provide a brief description of the adopted multi-wavelength data sets from various Galactic plane surveys (see also Table 1). In the present work, we selected a field of $\sim 27^{\prime} \times 20.5(\sim 29 \mathrm{pc} \times 22 \mathrm{pc})$; centered at $l=35^{\circ} .734 ; b=-0^{\circ} .914$.

\subsection{NIR Data}

We analyzed the photometric NIR data extracted from the United Kingdom Infra-Red Telescope (UKIRT) Infrared Deep Sky Survey (UKIDSS) Galactic Plane Survey (GPS; $\lambda=1.25,1.65,2.2 \mu \mathrm{m}$; Lawrence et al. 2007) sixth archival data release (UKIDSSDR6plus) and the 2MASS catalogs. The UKIDSS observations (resolution $\sim 0$ !' 8 ) were performed using the Wide Field Camera (WFCAM) at UKIRT. The calibration of UKIDSS fluxes was carried out using the 2MASS photometric data. In this work, we extract only reliable NIR photometric data from the catalog. To obtain reliable point sources from the GPS catalog, the conditions suggested by Lucas et al. (2008) are considered, which include the removal of saturated sources, non-stellar sources, and unreliable sources near the sensitivity limits. A SQL script (based on $\mathrm{Lu}-$ cas et al. 2008, and presented in Dewangan et al. (2015)) is especially written to implement these criteria. More information about the selection procedures of the GPS photometry can be found in Dewangan et al. (2015). We notice the UKIDSS saturation near $J=10.3, \mathrm{H}=$ 11.1 , and $\mathrm{K}=9.6 \mathrm{mag}$. Hence, in our final catalog, the magnitudes of the saturated bright UKIDSS sources were obtained from the 2MASS catalog. To select good 2MASS photometric quality data, we downloaded only those sources with a photometric magnitude error of 0.1 or less in each band.

\subsection{H-band Polarimetry}

To obtain background starlight polarization, NIR Hband $(1.6 \mu \mathrm{m})$ linear polarimetric data (resolution $\sim 1^{\prime \prime} .5$ ) are extracted from the Galactic Plane Infrared Polarization Survey (GPIPS; $\lambda=1.6 \mu \mathrm{m}$; Clemens et al. 2012a) Data Release 2 (i.e. DR2). These data sets were observed with the Mimir instrument, mounted on the 1.8 $\mathrm{m}$ Perkins telescope, in linear imaging polarimetry mode (see Clemens et al. 2012a for more details). To retrieve the most reliable polarimetric data, we selected the sources having $P / \sigma_{P} \geq 2, \sigma_{P} \leq 5$, and $H \leq 13 \mathrm{mag}$ (where $P$ is the polarization percentage and $\sigma_{p}$ is the polarimetric uncertainty).

\subsection{Spitzer and WISE Data}

We retrieve the photometric images and magnitudes of point sources at 3.6-8.0 $\mu \mathrm{m}$ from the Spitzer-GLIMPSE survey (resolution $\sim 2^{\prime \prime}$ ). The photometric data are extracted from the GLIMPSE-I Spring '07 highly reliable photometric catalog. We also use the Multiband Imaging Photometer for Spitzer (MIPS) Inner Galactic Plane Survey (MIPSGAL; $\lambda=24 \mu \mathrm{m}$; Carey et al. 2005) $24 \mu \mathrm{m}$ image (resolution $\sim 6^{\prime \prime}$ ) and the photometric magnitudes of point-like sources at $24 \mu \mathrm{m}$ (from Gutermuth \& Heyer 2015).

The archival Wide Field Infrared Survey Explorer (WISE ${ }^{1} \lambda=3.4,4.6,12,22 \mu \mathrm{m}$; Wright et al. (2010)) image at MIR $12 \mu \mathrm{m}$ (spatial resolution $\sim 6^{\prime \prime}$ ) is also retrieved.

\subsection{Herschel Data}

${ }^{1}$ WISE is a joint project of the University of California and the JPL, Caltech, funded by the NASA 
To probe the far-infrared (FIR) and sub-millimeter (sub-mm) emission, Herschel continuum maps at $70 \mu \mathrm{m}$, $160 \mu \mathrm{m}, 250 \mu \mathrm{m}, 350 \mu \mathrm{m}$, and $500 \mu \mathrm{m}$ are obtained from the Herschel Space Observatory (Pilbratt et al. 2010, Poglitsch et al. 2010, Griffin et al. 2010; de Graauw et al. 2010) data archives. Level2_5 processed Herschel images were downloaded through the Herschel Interactive Processing Environment (HIPE, Ott 2010). These observations are part of the Herschel Infrared Galactic Plane Survey (Hi-GAL; $\lambda=70,160,250,350,500 \mu \mathrm{m}$; Molinari et al. 2010) project. The plate scales of 70,160 , 250,350 , and $500 \mu \mathrm{m}$ images are $3^{\prime \prime} .2,3^{\prime \prime} .2,6^{\prime \prime}, 10^{\prime \prime}$, and $14^{\prime \prime}$ pixel $^{-1}$, respectively. The beam sizes of the Herschel images are $5^{\prime \prime} .8,12^{\prime \prime}, 18^{\prime \prime}, 25^{\prime \prime}$, and $37^{\prime \prime}$ for 70,160 , 250, 350, and $500 \mu \mathrm{m}$, respectively (Poglitsch et al. 2010. Griffin et al. 2010). The Herschel images at 70-160 $\mu \mathrm{m}$ are calibrated in $\mathrm{J}_{\mathrm{y}}$ pixel $^{-1}$, while the units of images at 250-500 $\mu \mathrm{m}$ are MJy sr ${ }^{-1}$.

Following the methods presented in Mallick et al. (2015), the Herschel temperature and column density maps are generated using the Herschel continuum images. These maps are produced from a pixel-by-pixel spectral energy distribution (SED) fit with a modified blackbody to the cold dust emission at Herschel 160-500 $\mu \mathrm{m}$ (see also Dewangan et al. 2015).

A brief step-by-step explanation of the adopted procedures is as follows. Before the SED fit, the 160-350 $\mu \mathrm{m}$ images are converted into the same flux unit (i.e. Jy pixel $^{-1}$ ) and convolved to the lowest angular resolution of the $500 \mu \mathrm{m}$ image $\left(\sim 37^{\prime \prime}\right)$. Furthermore, we regrid these images to the pixel size of the $500 \mu \mathrm{m}$ image $\left(\sim 14^{\prime \prime}\right)$. These steps are carried out using the plug-in "Photometric Convolution" and task "Convert Image Unit" available in the HIPE software. Next, the sky background flux level was computed to be $0.211,0.566,1.023$, and $0.636 \mathrm{Jy} \mathrm{pixel}^{-1}$ for the $500,350,250$, and $160 \mu \mathrm{m} \mathrm{im}-$ ages (size of the selected featureless dark region $\sim 4^{\prime} .9 \times$ 6 ' 4 ; centered at: $\left.l=35^{\circ} .208 ; b=-1^{\circ} .47\right)$, respectively.

In the final step, a modified blackbody is fitted to the observed fluxes on a pixel-by-pixel basis to obtain the temperature and column density maps (see equations 8 and 9 in Mallick et al. 2015). The fitting is performed using the four data points for each pixel, retaining the dust temperature $\left(\mathrm{T}_{d}\right)$ and the column density $\left(N\left(\mathrm{H}_{2}\right)\right)$ as free parameters. In the analysis, we use a mean molecular weight per hydrogen molecule $\left(\mu_{H 2}\right)$ of 2.8 (Kauffmann et al. 2008) and an absorption coefficient $\left(\kappa_{\nu}\right)$ of $0.1(\nu / 1000 \mathrm{GHz})^{\beta} \mathrm{cm}^{2} \mathrm{~g}^{-1}$, including a gas-to-dust ratio $\left(R_{t}\right)$ of 100, with a dust spectral index $(\beta)$ of 2 (see Hildebrand 1983). We further discuss these Herschel maps in Section 3.5

\subsection{Planck $353 \mathrm{GHz}$ data}

To obtain dust polarized emission, we use the Planch ${ }^{2}$ polarization data $(\nu=353 \mathrm{GHz}$; Planck Collaboration IX 2014) observed with the High Frequency Instrument (HFI) at $353 \mathrm{GHz}$, where the dust polarized emission is

2 Planck (http://www.esa.int/Planck) is a project of the European Space Agency (ESA) with instruments provided by two scientific consortia funded by ESA member states and led by Principal Investigators from France and Italy, telescope reflectors provided through a collaboration between ESA and a scientific consortium led and funded by Denmark, and additional contributions from NASA (USA). brightest. The Planck intensity and polarization (Stokes parameters $I, Q$, and $U$ ) sky maps are obtained from the 2015 release of Planck legacy archive (Planck Collaboration I 2016a). The procedures of map-making, calibration, and correction of systematic effects are described in Planck Collaboration VIII (2016b). We smooth the maps from an initial angular resolution of $4^{\prime} .9$ to $6^{\prime}$ to increase the signal-to-noise ratio and to minimize beam depolarization effects. The contribution from the cosmic microwave background (CMB) emission is very minimum and has been ignored. The intensity and polarization maps are converted from temperature scale of $K_{\mathrm{CMB}}$ to MJy sr ${ }^{-1}$ using the unit conversion and color correction factor of 246.543 (Planck Collaboration IX 2014). The intensity map is also subtracted from the Galactic zero level offset of $0.0885 \mathrm{MJy} \mathrm{sr}^{-1}$ (Planck Collaboration VIII 2016b). The linear polarization of dust emission integrated along the line-of-sight is measured from the Stokes parameters $I, Q$, and $U$ as:

$$
\begin{gathered}
P=\sqrt{Q^{2}+U^{2}} \\
p=100 * \frac{P}{I} \\
\psi=\frac{1}{2} \tan ^{-1}(U, Q) .
\end{gathered}
$$

where $P$ is the polarization intensity, $p$ is the percentage of dust polarization fraction, and $\psi$ is the polarization angle. The Planck Stokes parameters are in the HEALPix (Górski et al. 2005) convention having the angle $\psi=0$ toward the north Galactic pole and positive toward the west (clockwise). We use the IAU convention (Hamaker \& Bregman 1996) by changing the Planck Stokes $U$ map to negative so that the polarization angle is measured anticlockwise relative to the north Galactic pole. Since the noise in the maps introduces a positive bias on the measured polarization, we debias the polarization following the method given in Plaszczynski et al. (2014), and using the full available noise covariance matrix.

$$
\text { 2.6. }{ }^{13} \mathrm{CO}(\mathrm{J}=1-0) \text { Line Data }
$$

In our selected target region, we probe the molecular gas content in the MCG35.6 using the GRS ${ }^{13} \mathrm{CO}$ $(\mathrm{J}=1-0)$ line data. The GRS line data have a velocity resolution of $0.21 \mathrm{kms}^{-1}$, an angular resolution of $45^{\prime \prime}$ with $22^{\prime \prime}$ sampling, a main beam efficiency $\left(\eta_{\mathrm{mb}}\right)$ of $\sim 0.48$, a velocity coverage of -5 to $135 \mathrm{~km} \mathrm{~s}^{-1}$, and a typical rms sensitivity $(1 \sigma)$ of $\approx 0.13 \mathrm{~K}$ (Jackson et al. 2006).

\subsection{Radio Centimeter Continuum emission}

To trace the ionized gas emission in the G35.6 site, we use the NVSS $1.4 \mathrm{GHz}$ continuum map. The survey covers the sky north of $\delta_{2000}=-40^{\circ}$ at $1.4 \mathrm{GHz}$ with a beam of $45^{\prime \prime}$ and a nearly uniform sensitivity of $\sim 0.45$ mJy/beam (Condon et al. 1998). The Coordinated Radio and Infrared Survey for High-Mass Star Formation (CORNISH; Hoare et al. 2012) 5 GHz $(6 \mathrm{~cm})$ radio continuum data (beam size $\sim 1.5$ ) are also utilized in the present work. 


\section{RESULTS}

\subsection{Physical environment of G35.6}

The investigation of the ionized gas, dust (cold and warm), and molecular emission enables us to probe the embedded structures present in a given star-forming region. In Figure 1, we show the spatial distribution of dust (warm and cold), molecular gas, and ionized gas toward the G35.6 site. The radio continuum data trace the ionized gas emission, while the sub-mm data are sensitive to the cold dust. The MIR and FIR data trace the warm dust. Figure $1 \mathrm{a}$ is a three-color composite image made using the MIPSGAL $24 \mu \mathrm{m}$ in red, WISE $12 \mu \mathrm{m}$ in green, and GLIMPSE $5.8 \mu \mathrm{m}$ in blue. The color composite image is overlaid with the NVSS radio continuum emission at $1.4 \mathrm{GHz}$, revealing two compact radio sources (designated as crs1 and crs2). These radio sources, crs1 and crs2, were previously referred to as NVSS 185929+020334 and NVSS 185938+020012, respectively (e.g., Condon et al. 1998, Paron et al. 2011). One of the radio sources, crs1 is seen near the location of IRAS $18569+0159$. The inset on the bottom left shows a zoomed-in view of the two radio continuum peaks using the WISE and Spitzer images (12 $\mu \mathrm{m}$ (red), $8.0 \mu \mathrm{m}$ (green), and $5.8 \mu \mathrm{m}$ (blue); see Figure 1a). There is an extended emission seen at 5.8-12 $\mu \mathrm{m}$. In the literature, it has been reported that the ionized gas and the MIR/FIR emission (at $12 / 24 / 70 \mu \mathrm{m}$ ) are seen systematically correlated in H II regions (e.g. Deharveng et al. 2010, Paladini et al. 2012). In the vicinity of crs1 and crs2, infrared features are also noticeably observed (see broken circles in the inset). It is also known that the $5.8-12 \mu \mathrm{m}$ bands contain various polycyclic aromatic hydrocarbon (PAH) features at $6.2,7.7,8.6$, and $11.3 \mu \mathrm{m}$ (including the continuum). The presence of PAH features can be used to trace photodissociation regions (or photon-dominated regions, or PDRs) and enables us to infer the impact and influence of the H II regions on their surroundings (see broken circles in the inset). The detection of the CORNISH $5 \mathrm{GHz}$ continuum emission is also indicated by a square in Figure 1a (see also the inset). The quantitative calculations using these radio data are performed in Section 3.3 .

Figure $1 \mathrm{~b}$ shows a three-color composite image made using the Herschel $160 \mu \mathrm{m}$ (in red), Herschel $70 \mu \mathrm{m}$ (in green), and GLIMPSE $8.0 \mu \mathrm{m}$ (in blue) images. The composite image is overlaid with the ${ }^{13} \mathrm{CO}$ molecular gas emission. In the direction of our selected region, the ${ }^{13} \mathrm{CO}$ emission traces the molecular gas in the velocity range of $53-62 \mathrm{~km} \mathrm{~s}^{-1}$. The distribution of ${ }^{13} \mathrm{CO}$ also depicts a molecular shell. Furthermore, in the composite map, an almost semi-ring-like feature is prominently observed. As mentioned earlier, these ionized and molecular features are already known in the literature (e.g. Paron et al. 2011). In the composite map, a small dotted box (in white) highlights the area investigated by Paron et al. (2011). Additionally, a molecular condensation is also observed in the Galactic eastern side of the molecular shell (see Figure 1p). A detailed analysis of the molecular and ionized features is presented in Sections 3.2 and 3.3 , respectively.

Most of the molecular gas is spatially found in the Galactic southern side of the radio source, crs1 (see a yellow box in Figure 1b). A zoomed-in multi-wavelength view of this area (selected field $\sim 7.3 \times 99^{\prime} 6$ (or $\sim 7.8 \mathrm{pc}$ $\times 10.3 \mathrm{pc})$ ) is presented in Figure 2. The images are shown at $8.0 \mu \mathrm{m}, 12 \mu \mathrm{m}, 24 \mu \mathrm{m}, 70 \mu \mathrm{m}, 160 \mu \mathrm{m}, 250$ $\mu \mathrm{m}, 350 \mu \mathrm{m}, 500 \mu \mathrm{m}$, and integrated intensity map of ${ }^{13} \mathrm{CO}(\mathrm{J}=1-0)$ from 53 to $62 \mathrm{~km} \mathrm{~s}^{-1}$. Note that an almost ring-like feature is evident in the integrated ${ }^{13} \mathrm{CO}$ intensity map and the Herschel sub-mm images (at 250$500 \mu \mathrm{m})$. The central cavity of the molecular emission coincides with a dark region in the infrared images, suggesting that there are no stars and dust condensations. The integrated ${ }^{13} \mathrm{CO}$ intensity map and the image at 12 $\mu \mathrm{m}$ are also superimposed with the NVSS $1.4 \mathrm{GHz}$ emission, indicating that the ionized gas emission is not seen inside the ring-like feature. However, the radio source, crs2 is spatially seen at the most north-western section of the ring-like feature. In the closest vicinity of crs2, within a circle with a radius of $1.25 \mathrm{pc}$, we find a small feature seen in the infrared and sub-mm images (see a circle in Figure 2 and also the inset in Figure 1 1 ). The feature is resolved in the images below at $100 \mu \mathrm{m}$, while, in the longer wavelength images $(>100 \mu \mathrm{m})$, it becomes a part of the ring-like feature. The energetic and feedback of the massive star associated with crs2 seems to influence this feature (see Section 3.4 for more details).

The analysis of multi-wavelength images reveals the presence of a semi-ring-like feature (associated with the radio source crs1) and an embedded ring-like feature (without detectable ionized gas emission), at the sensitivity of the observed NVSS radio map ( $1 \sigma \sim 0.45 \mathrm{mJy}$ beam $\left.^{-1}\right)$ in the G35.6 site. The presence of a ring-like feature in G35.6 is an excellent example for comparing with theoretical models, since observationally it is scarce and difficult to find such features with a high degree of geometrical and projectional symmetry. Overall, with the help of Figures 11, and 2, we have obtained a pictorial multi-wavelength view of the G35.6 site.

\subsection{Molecular gas properties in MCG35.6}

In this section, we present a kinematic analysis of molecular gas in the MCG35.6. Note that previously, Paron et al. (2011) also analyzed the GRS ${ }^{13} \mathrm{CO}$ data and presented the integrated GRS ${ }^{13} \mathrm{CO}$ intensity map and velocity channel maps mainly toward the G35.6 H II region (see a small dotted box in Figure 1 b). In the present work, we have carefully examined the gas distribution in the direction of our selected target field, and have also performed the position-velocity analysis of molecular gas, which was lacking in the earlier published work.

\subsubsection{Velocity profiles and molecular cloud}

The integrated GRS ${ }^{13} \mathrm{CO}(\mathrm{J}=1-0)$ velocity channel maps (at intervals of $1 \mathrm{~km} \mathrm{~s}^{-1}$ ) are presented in Figure 3 . which cover the velocity range from 54 to $63 \mathrm{~km} \mathrm{~s}^{-1}$. The channel maps reveal two spatially distinct molecular structures, which can also be referred to as extended molecular condensations (EMCs). The first one (EMC1) is seen in a velocity range from 55 to $60 \mathrm{~km} \mathrm{~s}^{-1}$, and appears to be associated with the radio continuum emission. It has an extended morphology showing an almost ring-like feature at a velocity of $57-58 \mathrm{~km} \mathrm{~s}^{-1}$ (see also Figure 2). The second structure (EMC2) appears at velocities from 56 to $62 \mathrm{~km} \mathrm{~s}^{-1}$. At low velocities it is compact and peaks at the coordinates $l=35^{\circ} .85, b=$ 
$-0^{\circ} .94$, while it extends to the north when moving to high velocities having the red-shifted peak at the coordinates $\left(l=35^{\circ} .85, b=-0^{\circ} .85\right)$.

Figure 4 a shows an integrated intensity map of ${ }^{13} \mathrm{CO}$ $(\mathrm{J}=1-0)$ from 53 to $62 \mathrm{~km} \mathrm{~s}^{-1}$, revealing clearly both the molecular condensations, EMC1 and EMC2 (see also Figure 3). In the integrated ${ }^{13} \mathrm{CO}$ map, the ring-like feature is evident at the Galactic southern side of EMC1. In Figure $4 \mathrm{~b}$, we show the observed ${ }^{13} \mathrm{CO}(\mathrm{J}=1-0)$ spectral profiles in the direction of EMC1 and EMC2 (see respective box in Figure 4 a). In the direction of EMC1, the velocity peak is seen at $\sim 57 \mathrm{~km} \mathrm{~s}^{-1}$, while the velocity peak toward EMC2 is found at $\sim 60 \mathrm{~km} \mathrm{~s}^{-1}$. These profiles further confirm the presence of two molecular cloud components in our selected site. Figure 4 . shows the observed ${ }^{13} \mathrm{CO}(\mathrm{J}=1-0)$ spectra in the direction of six fields associated with the ring-like feature (i.e. zone- 2 to zone-7; see broken boxes in Figure 4 a). Each spectrum is constructed by averaging the area highlighted by a box in Figure 4 a. In Figure 4c, the velocity peaks in the profiles are varying between 57 and $58.5 \mathrm{~km} \mathrm{~s}^{-1}$. Based on the comparison of velocity peaks in six profiles, one can find evidence of gas motion in the molecular feature. One can note that zones 2, 4, and 6 trace the west segment of the ring-like feature, while zones 3,5 , and 7 depict the eastern side. The velocity peaks seen in the direction of zones 2,4 , and 6 have very similar values, while the velocity peaks observed in the direction of zones 3,5 , and 7 have slightly different values, and also have noticeable higher values (i.e. red-shifted) than the ones seen toward the zones 2, 4, and 6 (see Figure 4 ). This result also indicates the existence of a velocity spread across the ring-like feature (see also Section 3.2.2.

Considering these results and the spatial distribution of molecular gas, the ring-like feature has an ellipsoid shape along the line of sight. In 3D, we assume that this molecular feature has a spherical oblate morphology.

\subsubsection{Position-velocity diagrams}

To further study the molecular gas distribution in the direction of our selected target field, in Figure 5 . we present the integrated ${ }^{13} \mathrm{CO}$ intensity map and the position-velocity diagrams.

In Figure 5a, we show the molecular ${ }^{13} \mathrm{CO}(\mathrm{J}=1-0)$ gas emission in the direction of the G35.6 site, which is the same as shown in Figure 4a. In Figures $5 \mathrm{~b}$ and $5 \mathrm{~d}$, we present the Galactic position-velocity diagrams of ${ }^{-13} \mathrm{CO}$ emission. The ${ }^{13} \mathrm{CO}$ emission is integrated over the longitude range and latitude range in the latitude-velocity diagram (see Figure 5b) and longitude-velocity diagram (see Figure 5d), respectively. The position-velocity diagrams also trace both the molecular components along the line of sight. A molecular component associated with the G35.6 $\mathrm{H}$ II region is seen in a velocity range of $53-59 \mathrm{~km}$ $\mathrm{s}^{-1}$ (i.e. EMC1), while the other molecular component is observed at $59-62 \mathrm{~km} \mathrm{~s}^{-1}$ (i.e. EMC2) (see also Section 3.2.1). In Figure 5, we present the spatial distribution of molecular gas associated with these two molecular components. The figure also reveals that these two components (i.e., EMC1 and EMC2) are clearly separated in both space and velocity, and are unrelated molecular clouds. Hence, our present work is mainly focused toward EMC1.
Figures 5e, 5f, and 58 show the position-velocity diagrams of " ${ }^{13} \mathrm{O}$ along the axis "p1-dp1", "p2-dp2", and "p3-dp3", respectively. The axis "p1-dp1" is selected toward the radio source crs1, while the other two axes are chosen toward the ring-like feature. In the direction of crs1/the semi-ring-like feature, the position-velocity diagrams show hints of an almost inverted C-like structure (see Figure 5e and also a black dashed curve in Figure $5 \mathrm{~b}$ ). In the velocity space, the detection of an inverted $\mathrm{C}$-like or arc-like structure indicates the presence of a bubble/shell (Arce et al. 2011). Hence, in the direction of the semi-ring-like feature, our analysis suggests the presence of an expanding shell with an expansion velocity ${ }^{3}$ of the gas to be $\sim 1.2 \mathrm{~km} \mathrm{~s}^{-1}$. The position of crs1 is highlighted in Figures $5 a^{-}-5$, and nearly appears at the center of the inverted C-like structure (see Figures $5 \mathrm{~b}$ and 5 ).

In Figures $5 \mathrm{~b}$ and $5 \mathrm{~d}$, a noticeable velocity spread is also found, where the majority of molecular gas is observed, indicating the gas movement in the ring-like feature (see also Figure 4 .). In the direction of the ring-like feature, Figures 5 and 5 g further reveal the observed velocity structures. A velocity spread across the molecular feature is also evident in the diagrams. In the velocity space, two velocity peaks (i.e. 57 and $58 \mathrm{~km} \mathrm{~s}^{-1}$ ) are seen, likely connected by diffuse gas and therefore suggesting the presence of a single structure with velocity gradients. Higher spectral and angular resolution data can better resolve the structure and the kinematics of the gas in the ring-like structure. As mentioned earlier, there is no ionized gas emission seen inside the ring-like feature, hence the observed velocity spread may not be explained by an $\mathrm{H}$ II region.

\subsection{Ionized gas properties}

In this section, we investigate the spectral types of the powering candidates responsible for the compact radio sources (i.e., crs1 and crs2). We have computed the number of Lyman continuum photons $\left(N_{\mathrm{uv}}\right)$ for each compact radio source using the integrated flux density following the equation of Matsakis et al. (1976). The "clumpfind" IDL program (Williams et al. 1994) can be employed in a given radio continuum map to compute the integrated flux densities. Using the $1.4 \mathrm{GHz}$ map, we estimated the integrated flux densities (radii) equal to $160.7 \mathrm{mJy}(1.8$ pc) and $58.8 \mathrm{mJy}(1.25 \mathrm{pc})$ for compact radio sources, crs1 and crs2, respectively. The equation of $N_{\mathrm{uv}}$ is defined by Matsakis et al. (1976):

$$
\begin{aligned}
N_{u v}\left(s^{-1}\right)=7.5 \times 10^{46}\left(\frac{S_{\nu}}{J y}\right)\left(\frac{D}{k p c}\right)^{2} & \left(\frac{T_{e}}{10^{4} K}\right)^{-0.45} \\
& \times\left(\frac{\nu}{G H z}\right)^{0.1}
\end{aligned}
$$

where $\mathrm{S}_{\nu}$ is the measured total flux density in Jy, D is the distance in $\mathrm{kpc}, \mathrm{T}_{e}$ is the electron temperature, and $\nu$ is the frequency in $\mathrm{GHz}$. These calculations were carried out for a distance of $3.7 \mathrm{kpc}$ and for the electron temperature of $10000 \mathrm{~K}$. Finally, we find $N_{\mathrm{uv}}\left(\right.$ or $\left.\log N_{\mathrm{uv}}\right)$ to be $\sim 1.7 \times 10^{47} \mathrm{~s}^{-1}(47.24)$ and $\sim 6.3 \times 10^{46} \mathrm{~s}^{-1}$ (46.8) for

3 In the velocity space, the expansion velocity corresponds to half of the velocity range for the inverted C-like structure. 
compact radio sources, crs1 and crs2, respectively. Each compact radio source appears to be associated with a single ionizing star of spectral type B0.5V-B0V (see Table II in Panagia 1973, and also Smith et al. (2002)).

Paron et al. (2011) utilized a total flux density of 0.86 Jy at $2.7 \mathrm{GHz}$ for $\mathrm{G} 35.6$, and estimated $N_{\text {uv }}$ to be $\sim 1.0$ $\times 10^{48} \mathrm{~s}^{-1}$. The $2.7 \mathrm{GHz}$ continuum data, as part of the Bonn $11 \mathrm{~cm}$ survey, were taken with the Effelsburg 100 meter telescope, and have an angular resolution of $4^{\prime} .3$, and $50 \mathrm{mK}$ rms sensitivity (Reich et al. 1984). Note that the $2.7 \mathrm{GHz}$ continuum data have a much coarser resolution compared to the NVSS $1.4 \mathrm{GHz}$ continuum map. Hence, the total $2.7 \mathrm{GHz}$ flux density appears to contain radio emission contribution from both the radio sources, crs1 and crs2. Using the NVSS $1.4 \mathrm{GHz}$ data, the fluxes of crs1 and crs2 added together result in about $220 \mathrm{mJy}$. This value is much smaller than the total flux density at $2.7 \mathrm{GHz}$ for G35.6, indicating that the Effelsburg map might be detecting some extended emission that is filtered out by the interferometer in the NVSS survey. Furthermore, Paron et al. (2011) used the $1.4 \mathrm{GHz}$ data for the compact radio source, crs2 (or NVSS 185938+020012), and computed $N_{\text {uv }}$ to be $\sim 0.6 \times 10^{47} \mathrm{~s}^{-1}$, which is consistent with our estimates. Using these values of $N_{\mathrm{uv}}$, we find at our end that these estimates correspond to a single ionizing star of spectral type $09 \mathrm{~V}-08.5 \mathrm{~V}$ and B0.5V-B0V for G35.6 and crs2 (see Martins et al. (2005), Panagia (1973), and Smith et al. (2002), for theoretical values), respectively. However, Paron et al. (2011) reported the spectral type of the ionizing star of G35.6 to be between $\mathrm{O} 7.5 \mathrm{~V}$ and O9V , considering errors of about ten percent in both the distance and the radio continuum flux at $2.7 \mathrm{GHz}$. They also reported the spectral type of the ionizing star of crs2 to be later than $09.5 \mathrm{~V}$ star. These authors have not computed the radio spectral type for crs1. Based on our independent calculations using the $1.4 \mathrm{GHz}$ data, it appears that the spectral types of radio sources reported by Paron et al. (2011) are overestimated. Hence, spectroscopic observations will be needed to confirm the spectral types of the exciting stars. Using the photometric data, Paron et al. (2011) suggested that a source $\mathrm{J} 18592786+0203057$ is the more likely exciting-star candidate of G35.6, while two objects J18593584+0200579 and J18593556+0200488 could be the powering source candidates of crs2 (see Table 1 in their paper). Furthermore, in the G35.6 site, a point-like source is also observed in the CORNISH $5 \mathrm{GHz}$ continuum map (beam size $\sim 1^{\prime \prime} .5$ ), and is referred to as G035.6624-00.8481 in the CORNISH $5 \mathrm{GHz}$ compact source catalog (Purcell et al. 2013). The position of this radio source is indicated by a square in Figure 1 a, and appears about $1^{\prime} .4$ away from the peak position of crs1. However, in the direction of crs1, the CORNISH source is seen well within the NVSS radio emission contours (see Figure 1 a). Purcell et al. (2013) also computed a total integrated flux density equal to $5.43 \mathrm{mJy}$ for the CORNISH source (having an angular size of $\sim 1^{\prime} .5$ ). With the help of equation 4 , we find that the observed $5 \mathrm{GHz}$ flux (having $\log N_{\mathrm{uv}} \sim 45.82$ $\mathrm{s}^{-1}$ ) of G035.6624-00.8481 can be explained by a single ionizing star of spectral type B1V-B0.5V, which is nearly consistent with the spectral type of crs1 estimated using the NVSS $1.4 \mathrm{GHz}$ data.
With the estimates of $N_{\text {uv }}$ and radii of the H II regions $\left(\mathrm{R}_{H I I}\right)$, we have also computed the dynamical age $\left(\mathrm{t}_{d y n}\right)$ of each compact radio source. The age of the H II region can be obtained at a given radius $\mathrm{R}_{H I I}$, using the following equation (Dyson \& Williams 1980):

$$
t_{d y n}=\left(\frac{4 R_{s}}{7 c_{s}}\right)\left[\left(\frac{R_{H I I}}{R_{s}}\right)^{7 / 4}-1\right]
$$

where $c_{s}$ is the isothermal sound velocity in the ionized gas $\left(\mathrm{c}_{s}=11 \mathrm{~km} \mathrm{~s}^{-1}\right.$; Bisbas et al. (2009)), $\mathrm{R}_{H I I}$ is previously defined, and $\mathrm{R}_{s}$ is the radius of the Strömgren sphere $\left(=\left(3 N_{\mathrm{uv}} / 4 \pi n_{0}^{2} \alpha_{B}\right)^{1 / 3}\right.$, where the radiative recombination coefficient $\alpha_{B}=2.6 \times 10^{-13}\left(10^{4} \mathrm{~K} / \mathrm{T}\right)^{0.7}$ $\mathrm{cm}^{3} \mathrm{~s}^{-1}$ (Kwan 1997), $N_{\mathrm{uv}}$ is defined earlier, and " $\mathrm{n}_{0}$ " is the initial particle number density of the ambient neutral gas. Adopting a typical value of $\mathrm{n}_{0}\left(=10^{3} \mathrm{~cm}^{-3}\right)$, the dynamical ages of the compact radio sources, crs1 and crs 2 are estimated to be $\sim 0.5$ and $\sim 0.35$ Myr, respectively. Note that the H II regions are assumed to be uniform and spherically symmetric. Recently, Peters et al. (2010) indicated that the dynamical process of the H II regions could be more complex, and dynamical ages can not be estimated accurately with the assumptions of uniform and spherically symmetric. Hence, the derived dynamical ages of the $\mathrm{H}$ II regions can be considered as the indicative values.

The radio continuum map at $1.4 \mathrm{GHz}$ is also utilized to obtain the electron density $\left(n_{e}\right)$ of the ionized gas. The formula of " $n_{e}$ " is presented in Panagia \& Walmsley (1978) under the assumption that the H II region has a roughly spherical geometry:

$$
\begin{array}{r}
n_{e}\left(\mathrm{~cm}^{-3}\right)=3.113 \times 10^{2}\left(\frac{S_{\nu}}{\mathrm{Jy}}\right)^{0.5}\left(\frac{T_{e}}{10^{4} \mathrm{~K}}\right)^{0.25}\left(\frac{D}{\mathrm{kpc}}\right)^{-0.5} \\
\times b(\nu, T)^{-0.5} \theta_{R}^{-1.5}
\end{array}
$$

In the equation above, $\mathrm{S}_{\nu}, T_{e}$, and $\mathrm{D}$ are defined as in Equation $4, \theta_{R}$ is the angular radius in arcminutes, and

$b(\nu, T)=1+0.3195 \log \left(\frac{T_{e}}{10^{4} \mathrm{~K}}\right)-0.2130 \log \left(\frac{\nu}{1 \mathrm{GHz}}\right)$.

Considering the values of $\mathrm{S}_{\nu}$ and $\theta_{R}$ for each of the compact radio sources, the values of $n_{e}$ are computed to be 30 and $32 \mathrm{~cm}^{-3}$ for crs 1 and crs2, respectively. Using the CORNISH $5 \mathrm{GHz}$ data, we have also computed the value of $n_{e}$ to be $9250 \mathrm{~cm}^{-3}$ for G035.6624-00.8481. Hence, the CORNISH $5 \mathrm{GHz}$ observations suggest the presence of an ultracompact $\mathrm{H}$ II region in the G35.6 site. The calculation was carried out for $\mathrm{D}=3.7 \mathrm{kpc}$ and $\mathrm{T}_{e}=$ $10000 \mathrm{~K}$. Note that the electron density of these two radio sources estimated using the $1.4 \mathrm{GHz}$ data appears smaller than the one measured assuming optically thin emission. Therefore, radio continuum observations with radio interferometers at different frequencies can be helpful to better determine the SED of the $\mathrm{H}$ II regions and obtain a more accurate measurement of the properties of the HII regions and the spectral type of the ionizing stars (e.g. Panagia \& Felli 1975, Olnon 1975; Kurtz 2005, Sánchez-Monge et al. 2013).

\subsection{Feedback effect of massive stars}


Our radio data analysis reveals that each compact radio source is excited by a radio spectral type of $\mathrm{B} 0.5 \mathrm{~V}$ star (see Section 3.3). The radio continuum peak of crs1 is found to be associated with the semi-ring-like structure (see Figure 1a). It is also observed that the radio source crs2 is located toward the north-western part of the ring-like feature (see Figure 2). To explore the feedback of the $\mathrm{B} 0.5 \mathrm{~V}$ star in its vicinity, we infer the values of three pressure components (i.e., pressure of an H II region $\left(P_{\mathrm{HII}}\right)$, radiation pressure $\left(P_{\mathrm{rad}}\right)$, and stellar wind ram pressure $\left.\left(P_{\text {wind }}\right)\right)$ driven by a massive star. The equations of these pressure components $\left(P_{\mathrm{HII}}, P_{\mathrm{rad}}\right.$, and $\left.P_{\text {wind }}\right)$ are given below (e.g. Bressert et al. 2012):

$$
\begin{gathered}
P_{H I I}=\mu m_{H} c_{s}^{2}\left(\sqrt{\frac{3 N_{u v}}{4 \pi \alpha_{B} D_{s}^{3}}}\right) ; \\
P_{r a d}=L_{b o l} / 4 \pi c D_{s}^{2} ; \\
P_{\text {wind }}=\dot{M}_{w} V_{w} / 4 \pi D_{s}^{2} ;
\end{gathered}
$$

where $N_{\mathrm{uv}}, c_{\mathrm{S}}$, and $\alpha_{\mathrm{B}}$ are previously defined, $\mu=0.678$ (in the ionized gas; Bisbas et al. 2009), $m_{\mathrm{H}}$ is the hydrogen atom mass, $M_{w}$ is the mass-loss rate, $\mathrm{V}_{w}$ is the wind velocity of the ionizing source, $L_{\mathrm{bol}}$ is the bolometric luminosity of the source, and $\mathrm{D}_{s}$ is the projected distance from the position of the B0.5V star where the pressure components are evaluated. The pressure components driven by massive stars are estimated at angular sizes of the radio sources (i.e. almost twice the radii of crs1 and crs2). Using the $1.4 \mathrm{GHz}$ map, we obtained the radii equal to $1.8 \mathrm{pc}$ and $1.25 \mathrm{pc}$ for radio sources, crs1 and crs2, respectively. Hence, we have computed the pressure components at $\mathrm{D}_{s}=3.5 \mathrm{pc}(2.5 \mathrm{pc})$ for crs1 (crs2).

In the calculations, we have adopted the values of $L_{\mathrm{bol}}$ $=19950 \mathrm{~L}_{\odot}\left(\right.$ Panagia 1973), $\dot{M}_{w}=2.5 \times 10^{-9} \mathrm{M}_{\odot} \mathrm{yr}^{-1}$ (Oskinova et al. 2011), and $V_{\mathrm{w}}=1000 \mathrm{~km} \mathrm{~s}^{-1}$ (Oskinova et al. 2011), for a B0.5V star. With the help of equations 4,5 , and 6 , in the case of crs 1 with $\mathrm{D}_{s}=3.5 \mathrm{pc}$, we find $P_{\mathrm{HII}} \approx 1.5 \times 10^{-11}$ dynes $\mathrm{cm}^{-2}, P_{\mathrm{rad}} \approx 1.7 \times 10^{-12}$ dynes $\mathrm{cm}^{-2}$, and $P_{\text {wind }} \approx 1.1 \times 10^{-14}$ dynes $\mathrm{cm}^{-2}$. This also gives a total pressure $\left(P_{\text {total }}=P_{\text {HII }}+P_{\text {rad }}+P_{\text {wind }}\right)$ driven by a massive star to be $\sim 1.7 \times 10^{-11}$ dynes $\mathrm{cm}^{-2}$. Similarly, in the case of crs 2 with $\mathrm{D}_{s}=2.5 \mathrm{pc}$, we obtain $P_{\text {HII }} \approx 1.5 \times 10^{-11}$ dynes $\mathrm{cm}^{-2}, P_{\text {rad }} \approx 3.4 \times 10^{-12}$ dynes $\mathrm{cm}^{-2}, P_{\text {wind }} \approx 2.1 \times 10^{-14}$ dynes $\mathrm{cm}^{-2}$, and $P_{\text {total }}$ $\approx 1.8 \times 10^{-11}$ dynes $\mathrm{cm}^{-2}$. These calculations suggest that in both cases, the pressure of the $\mathrm{H}$ II region is relatively higher than the radiation pressure and the stellar wind pressure. Note that in both the cases, $P_{\text {total }}$ is comparable to the pressure of a typical cool molecular cloud $\left(P_{\mathrm{MC}} \sim 10^{-11}-10^{-12}\right.$ dynes $\mathrm{cm}^{-2}$ for a temperature $\sim 20$ $\mathrm{K}$ and particle density $\sim 10^{3}-10^{4} \mathrm{~cm}^{-3}$ ) (see Table 7.3 of Dyson \& Williams 1980). This implies that the edges of the semi-ring and ring-like features are not wiped out by the impact of the ionized gas. Our analysis further suggests that the semi-ring-like morphology can be explained due to the ionizing feedback from the B0.5V type star, and the most north-western section of the ring-like feature (see a circle in Figure 2) is likely to be affected by the radio source crs2. Further observations of PDR tracers (e.g. Hollenbach \& Tielens 1997; Treviño-Morales et al. 2016; Goicoechea et al. 2017) can better confirm the influence of crs2 on the molecular gas of the ring-like feature.

\subsection{Temperature and column density maps of G35.6}

In this section, to probe the embedded structure and dust condensations, we present the Herschel temperature and column density maps (resolution $\sim 37^{\prime \prime}$ ) of the MCG35.6. The temperature and column density maps are shown in Figures $6 a$ and 6b, respectively. The procedures for producing the Herschel temperature and column density maps were described in Section 2.4.

In the Herschel temperature map, the compact radio continuum sources (i.e., crs1 and crs2) are seen associated with warmer emission $\left(T_{\mathrm{d}} \sim 22-27 \mathrm{~K}\right.$; see Figure $\left.6 \mathrm{a}\right)$, confirming the influence of the radio continuum sources on the dense gas as discussed in Section 3.4. In the Herschel column density map, several condensations are observed in the MCG35.6 (see Figure6b). The "clumpfind" IDL program is executed to identify the clumps and to estimate their total column densities. Several column density contour levels were used as an input parameter for the "clumpfind", and the lowest contour level was considered at $3 \sigma$. Nine clumps are found in the MCG35.6 and are highlighted in Figure 6r. Furthermore, the boundary of each clump is also presented in Figure 6r. With the knowledge of the total column density of each clump, we have also determined the mass of each Herschel clump using the following equation:

$$
M_{\text {clump }}=\mu_{H_{2}} m_{H} \text { Area }_{\text {pix }} \Sigma N\left(H_{2}\right)
$$

where $\mu_{H_{2}}$ is assumed to be 2.8 , Area $a_{p i x}$ is the area subtended by one pixel, and $\Sigma N\left(\mathrm{H}_{2}\right)$ is the total column density. The mass of each Herschel clump is provided in Table 2 The table also gives an effective radius and a peak column density of each clump, which are extracted from the clumpfind algorithm. The clump masses vary between $175 \mathrm{M}_{\odot}$ and $3390 \mathrm{M}_{\odot}$. The peak column densities of these clumps vary between $\sim 4.2 \times 10^{21} \mathrm{~cm}^{-2}\left(\mathrm{~A}_{V}\right.$ $\sim 4.5 \mathrm{mag}$ ) and $\sim 10 \times 10^{21} \mathrm{~cm}^{-2}\left(\mathrm{~A}_{V} \sim 10.5 \mathrm{mag}\right)$ (see Table 2 and also Figure $2 \mathrm{p}$ ). Here, we used a relation between optical extinction and hydrogen column density (i.e. $A_{V}=1.07 \times 10^{-21} N\left(\mathrm{H}_{2}\right)$; Bohlin et al. 1978). We find that the EMC1 contains five Herschel clumps (i.e., nos. 1, 2, 3, 4, and 5) and the total mass of these five clumps is $\sim 5535 \mathrm{M}_{\odot}$. Interestingly, the ring-like feature embedded within EMC1 is also traced in the Herschel column density map (see a solid yellow box in Figure $6 \mathrm{~b}$ ). Furthermore, at least five clumps (i.e., nos. 1, 2, 3, 4, and 5) appear to be nearly regularly spaced along the ringlike feature. The mean separation between the clumps is computed to be $164^{\prime \prime} .6 \pm 66^{\prime \prime} .5$ (or $2.95 \pm 1.20 \mathrm{pc}$ ). One can also find at least three Herschel clumps (i.e., nos. 6, 7, and 8) toward EMC2.

\subsection{Polarimetric properties of MCG35.6}

In this section, we analyze the background starlight polarization from the GPIPS and the dust polarized emission from the Planck, to study the magnetic field morphology and properties. The POS magnetic field direction can be inferred through the polarization observations from and due to dust grains. Polarization of radiation emitted by spinning dust grains is parallel to their 
long axis and the projected field direction can be obtained through the polarization vectors rotated by 90 degrees. The physical process (i.e. radiative torque mechanism) concerning the alignment of dust gains and magnetic field is described in Dolginov \& Mitrofanov (1976) (see also Lazarian 2007, Andersson et al. 2015). Using the polarization vectors of background stars, one can also obtain the field direction in the POS parallel to the direction of polarization (Davis \& Greenstein 1951). Polarization of background starlight can be explained due to the dichroism by non-spherical dust grains.

\subsubsection{Magnetic field structure}

To probe the magnetic field morphology using starlight polarimetry, it is important to examine the relative polarization position angle orientations of the background stars with respect to the molecular cloud. In Section 2 we mentioned about the selection conditions to obtain the sources with reliable polarimetric information. These selected sources were further exposed to a color condition of $\mathrm{J}-\mathrm{H}>1$ to depict the background stars that can successfully trace the POS magnetic field direction. A total of 462 stars have been selected in our target region around the G35.6 site. Figure $7 \mathrm{a}$ shows the observed H-band polarization of background starlight overlaid on the integrated molecular map. In Figure $7 \mathrm{a}$, the degree of polarization is depicted by the length of a vector, whereas the angle of a vector shows the polarization Galactic position angle. In Figure 7 a, in EMC1, we have also highlighted four subregions (i.e. R1, R2, R3, and R4) by boxes and each box has a size of $\sim 2^{\prime} .4 \times 2^{\prime} .4$. These subregions are associated with the areas of high column density in the Herschel column density map (see also Figure 6b).

In Figure $7 \mathrm{~b}$, we show the Planck polarization vectors (in blue) from the dust emission at $353 \mathrm{GHz}$. The vectors are rotated by $90^{\circ}$ to show the POS magnetic field direction. The degree of polarization from the dust emission observed in the POS also depends on the variations of magnetic field along the line-of-sight (LOS). This causes de-polarization effects in the measurement. Since we are studying only the magnetic field direction using the Planck data, we average all the polarization values across the region, resulting in equal values in the degree of polarization. This exercise leads to the same length for all the vectors, keeping their original position angles. The Planck data at $353 \mathrm{GHz}$ have a beam size of $5^{\prime}$ with a pixel scale of $1^{\prime} .5(\sim 1.6 \mathrm{pc}$ at a distance of $3.7 \mathrm{kpc}$ ). In order to compare the magnetic field direction from the $353 \mathrm{GHz}$ dust emission polarimetry and the NIR polarimetry, we calculate the mean polarization of the GPIPS data by spatially averaging the values matching the same pixel scale of the Planck data. The GPIPS polarization data are gridded into maps with box regions of $1^{\prime} .5 \times 1^{\prime} .5$. All the Stokes $Q$ and $U$ values within each box region are combined by variance-weighted mean with their corresponding errors. The mean stokes values for each region are then used to compute the Ricean corrected de-biased polarization percentages and position angles for each box region (e.g. Clemens et al. 2012b). The mean GPIPS polarization values are shown in red, along with the Planck polarization vectors (in blue) in Figure $7 \mathrm{~b}$.

In Figure 7b, the Planck and GPIPS polarization vec- tors are in agreement with their relative position angles. However, one can notice differences in the orientation of the Planck and GPIPS vectors toward the molecular condensations (or areas of high column density). The Planck data show higher SNR results toward the molecular condensations due to the strong dust emission, whereas the GPIPS data toward the molecular condensations have a few measurements with low SNR due to high extinction. In EMC1, the distribution of polarization vectors appears different toward the ring-like feature and the semiring-like structure, implying a change in the magnetic field morphology between the two features.

Figures $8 \mathrm{a}$ and $8 \mathrm{~b}$ show a zoomed in view of the ringlike feature using the Herschel column density and integrated molecular maps, respectively. These maps are also overlaid with the GPIPS H-band polarization vectors (having $P / \sigma_{P} \geq 2, \sigma_{P} \leq 5, H \leq 13$ and $J-H>1$ ) and the Herschel clumps. A total of 17 stars are detected toward the ring-like feature, tracing the POS magnetic field direction. Table 3 lists the 2MASS photometric magnitudes, degree of polarization, and polarization Galactic position angles of these 17 stars. The degree of polarization for the 17 sources varies between $\sim 2$ to $6 \%$, whereas the polarization Galactic position angles have a dispersion of around $25^{\circ}$ (see Table 3). With the help of the molecular gas distribution and the polarization data, we can characterize the orientation of polarization position angles with respect to the semi-major axis of the molecular structure. Fitting an ellipse to the ring-like feature (see Figure 8p), we find that the angle of the major axis of the ellipse is about $10^{\circ}$. The mean polarization angles in three subregions R1, R2, and R3 are computed to be about $175^{\circ}, 177^{\circ}$, and $169^{\circ}$, respectively, and are measured from North-up, counter-clockwise (see Figure $7 \mathrm{a}$ ). The difference between the polarization angle and the ellipse major axis is about $15^{\circ}$. This indicates that the POS magnetic field is nearly parallel to the major axis of the ring-like feature. In Figures $7 \mathrm{a}$ and $7 \mathrm{~b}$, the semiring-like feature is seen at the northern part of EMC1. Similarly, we fit a semi-ellipse to the semi-ring-like feature and obtain the angle of the major axis to be $150^{\circ}$. The mean polarization angle in the subregion R4 linked with the semi-ring-like feature is about $55^{\circ}$. The difference between the polarization angle and the major axis is about $95^{\circ}$, indicating that the POS field orientation is perpendicular to the molecular structure associated with the subregion R4.

\subsubsection{Magnetic field strength}

The POS magnetic field strength can be computed using the observed polarimetric data, in combination with gas motions and density from the molecular line data, following the equation given in Chandrasekhar \& Fermi (1953) (hereafter CF-method). The CF-method as modified by Ostriker et al. (2001) can be presented in the following manner:

$$
B_{\text {pos }}=0.5 \times\left(\frac{4}{3} \pi \rho\right)^{0.5} \times \frac{\sigma_{v}}{\alpha}
$$

where $B_{\text {pos }}$ is the POS magnetic field strength (in $\mu \mathrm{G}$ ), $\alpha$ is the polarization position angle (P.A.) dispersion (in radians), $\rho$ is the gas volume mass density (in $\mathrm{g} \mathrm{cm}^{-3}$ ), and $\sigma_{v}$ is the ${ }^{13} \mathrm{CO}$ gas velocity dispersion (in $\mathrm{cm} \mathrm{s}^{-1}$ ). 
Ostriker et al. (2001) also suggested that the modified CF-method is applicable only when P.A. dispersion values are within $25^{\circ}$ (i.e. $\alpha \leq 25^{\circ}$ ).

To measure the magnetic field strength in the denser subregions within EMC1, we have carefully chosen four subregions (R1-R4) and the size of each subregion is $2^{\prime} .4 \times 2^{\prime} .4$ (as highlighted by boxes in Figure 7 a). These subregions are centered around the Herschel clumps/molecular condensations. They are small enough to probe the magnetic field strength to few parsec scales and also big enough to have sufficient high quality polarization stars to measure the P.A. dispersion accurately.

The P.A. dispersion $(\alpha)$ is calculated by finding the standard deviation of the P.A. values within each box region. Since the P.A. values have an ambiguity of $0^{\circ}$ and $180^{\circ}$, dealiasing is necessary. This is done by finding the minimum of the dispersion values obtained for different sets of biased P.A. values. The biasing is carried out by stepping the P.A. values through increments of $1^{\circ}$ between $0^{\circ}$ and $180^{\circ}$ producing different sets of biased P.A. values. The standard deviation for each set is computed and the minimum of the different standard deviation values is chosen as the final dispersion value. Note that typically, five polarization vectors are needed to obtain an accurate value of the P.A. dispersion. In the present case, we have only three polarization vectors in the subregion R3, where the derived P.A. dispersion is about $17^{\circ}$. Considering this dispersion value, we can obtain a lower limit of the B-field in the subregion R3.

The gas velocity dispersion $\left(\sigma_{v}\right)$ is computed from the ${ }^{13} \mathrm{CO}$ line profile for each pixel within the subregion. The line profile is fitted with a Gaussian to obtain the velocity dispersion as the width of the Gaussian. Multiple dispersion values within our selected box regions are then combined by median filtered mean to obtain the average dispersion value to be used in the CF-method for each region. A mean spectrum toward each subregion is presented in Figure 9

The Herschel column density map is used to determine the volume densities for the four subregions in EMC1. Based on the ring-like structure assuming a face-on view, we assume a spherically oblate morphology for the cloud structure. Using this morphology we estimate the cloud thickness to be $\sim 4$ pc. Based on the cloud thickness and the column densities, we calculate the volume number density of the region. The volume densities were gridded as a map having a spatial resolution of $14^{\prime \prime}$. All the volume densities within our each selected box region are then averaged to obtain the mean volume density. This is then converted into volume mass density $\rho$ by taking into account the molecular hydrogen's weight $(2 \times 1.00794 \times$ $1.67 \times 10^{-24} \mathrm{gm}$ ) and a factor of 1.36 to account for helium and heavier elements. With the application of the CF-method, the POS magnetic field strength in each subregion has been estimated (see Table 4). Using the values of $B_{\text {pos }}$, we have also estimated magnetic pressure, $P_{\text {mag }}\left(=B_{\text {pos }}^{2}(G) / 8 \pi ;\right.$ dynes $\left.\mathrm{cm}^{-2}\right)$, equal to $\sim 1.8$ $\times 10^{-11}, \sim 4.8 \times 10^{-12}, \sim 4.8 \times 10^{-12}$, and $\sim 1.6 \times 10^{-11}$ dynes $\mathrm{cm}^{-2}$ for the subregions $\mathrm{R} 1, \mathrm{R} 2$, R3, and R4, respectively. The average magnetic field strength for all the subregions is computed to be about $15 \mu \mathrm{G}$ and the corresponding magnetic pressure is estimated to be $\sim 8.9$ $\times 10^{-12}$ dynes $\mathrm{cm}^{-2}$.
We have also obtained the total field strength by assuming equipartition of magnetic, gravitational, and kinetic energy using a relation given in Lada et al. (2004) (i.e. $B_{\mathrm{EP}}=\Delta \mathrm{V}_{N T} \times(3 \pi \rho / 2 \ln 2)^{1 / 2}$; where $\Delta \mathrm{V}_{N T}$ is the nonthermal component of the observed line width). The non-thermal velocity dispersion is given by:

$$
\Delta V_{\mathrm{NT}}=\sqrt{\frac{\Delta V^{2}}{8 \ln 2}-\frac{k T}{29 m_{H}}}=\sqrt{\frac{\Delta V^{2}}{8 \ln 2}-\Delta V_{\mathrm{T}}^{2}},
$$

where $\Delta V\left(=\left(8 \ln 2{\sigma_{v}}^{2}\right)^{1 / 2}\right)$ is the measured full width at half maximum (FWHM) of the observed ${ }^{13} \mathrm{CO}$ spectra, $\Delta V_{\mathrm{T}}\left(=\left(k T / 29 m_{H}\right)^{1 / 2}\right)$ is the thermal broadening for ${ }^{13} \mathrm{CO}$ at $\mathrm{T}$, and $m_{H}$ is the mass of the hydrogen atom. In the Herschel temperature map, the ring-like feature is depicted in a temperature range of about $18-20 \mathrm{~K}$. Using the values of $\Delta V$ and $\Delta V_{\mathrm{T}}$ (at $\mathrm{T}=20 \mathrm{~K}$; see Section 3.5), we derive $\Delta V_{N T}$, and then the values of $\mathrm{B}_{E P}$ are estimated to be $9.5,8.8,8.8$, and $6.3 \mu G$ for subregions $\mathrm{R} 1, \mathrm{R} 2, \mathrm{R} 3$, and $\mathrm{R} 4$, respectively.

The values of $\mathrm{B}_{E P}$ are slightly lower than that of $B_{\text {pos }}$. In general, an equipartition stage indicates that all the forces are equal. However, based on the observations, we find that the magnetic field may have played a dominant role in the cloud formation and its evolution.

\subsubsection{Mass to Flux Ratio}

The knowledge of the magnetic field strength and "mass-to-flux $(\mathrm{M} / \Phi)$ " ratio can enable to infer the magnetically subcritical and supercritical clouds/cores. These parameters can also allow to observationally assess the molecular clouds/cores stability and can help to understand the ongoing physical process in the cloud. However, the observational measurement of $M / \Phi$ of a cloud core is a difficult experiment (e.g. Clemens et al. 2016, and references therein).

The normalized mass-to-flux ratio $\left(\overline{M / \Phi_{B}}\right)$ can be calculated from magnetic field strength as follows (Crutcher et al. 2004):

$$
\overline{M / \Phi_{B}}=7.6 \times 10^{-21} \frac{N_{H_{2}}}{|B|}
$$

where $N_{H_{2}}$ is the column density (in $\mathrm{cm}^{-2}$ ) and $|B|$ is the 3-Dimensional magnetic field strength (in $\mu \mathrm{G}$ ). Note that in equation $13,|B|$ refers to the 3D magnetic field strength while our estimates are the POS magnetic field strength. Assuming that the LOS magnetic field is zero then one can replace $|B|$ with $B_{\text {pos }}$. However, in this case, $B_{\text {pos }}$ will represent a lower limit to the total field strength. Hence, the mass-to-flux ratios of the four subregions are upper limit values. To obtain the corrected normalized mass-to-flux ratio, Planck Collaboration XXXV (2016c) report a correction factor of $1 / 3$ and $3 / 4$ for cloud geometries having magnetic field perpendicular and parallel to their major axis, respectively. In the ring-like feature, the magnetic field is parallel to its major axis. Hence, we applied a correction factor of $3 / 4$ to the mass-to-flux ratio calculation for subregions $\mathrm{R} 1, \mathrm{R} 2$, and R3. In the semi-ring-like feature, the magnetic field is perpendicular to its major axis, favouring a correction factor of $1 / 3$ for computing the mass-to-flux ratio in the subregion $\mathrm{R} 4$. 
Using the POS magnetic field strength and the Herschel column density map, we have calculated the normalized mass-to-flux ratio for four subregions in EMC1. Three subregions (R1-R3) in EMC1 have mass-to-flux ratio greater than 1, implying the cloud is supercritical, while the subregion R4 is subcritical due to mass-to-flux ratio less than 1 . In the subcritical region, the magnetic field dominates over gravity. On the other hand, in the supercritical region, the magnetic field cannot prevent gravitational collapse and hence the cloud can evolve and fragment into denser cores. Table 4 lists the magnetic properties for all the four subregions (R1-R4) in EMC1.

\subsection{Young stellar population in MCG35.6}

\subsubsection{Selection of YSOs}

In this section, to identify embedded YSOs within the MCG35.6, we have utilized the photometric data at 1-24 $\mu \mathrm{m}$ extracted from various infrared photometric surveys (e.g., MIPSGAL, GLIMPSE, UKIDSS-GPS, and 2MASS). These data sets enable us to infer infrared excess sources. The infrared excess emission around YSOs is explained by the presence of an envelope and/or circumstellar disk. Previously, Paron et al. (2011) also presented 36 YSOs identified using the 2MASS and GLIMPSE 3.6-8.0 $\mu \mathrm{m}$ photometric data sets, which were restricted only within the EMC1 (see Figure 6 and Table 2 in Paron et al. 2011). In the present work, we have utilized the UKIDSS-GPS NIR data, in combination with the GLIMPSE 3.6-8.0 $\mu \mathrm{m}$ and MIPSGAL $24 \mu \mathrm{m}$ data for depicting infrared excess sources. Note that the UKIDSS-GPS NIR survey is three magnitudes deeper than 2MASS. Hence, our present analysis will allow us to trace more deeply embedded and faint YSOs compared to previously published work. A brief description of the selection of YSOs is as follows.

1. Using the Spitzer 3.6 and $24 \mu \mathrm{m}$ photometric data, Guieu et al. (2010) used a color-magnitude plot $([3.6]-[24] /[3.6])$ to identify the different stages of YSOs (see also Rebull et al. 2011, Dewangan et al. 2015. Dewangan 2017b). The color-magnitude plot is also utilized to distinguish the boundary of possible contaminants (i.e. galaxies and disk-less stars) against YSOs (see Figure 10 in Rebull et al. 2011). We have adopted this scheme in this work and the color-magnitude plot is shown in Figure 10a. Following the conditions adopted in Guieu et al. (2010) and Rebull et al. (2011), the boundaries of different stages of YSOs and possible contaminants are highlighted in Figure $10 \mathrm{a}$. In Figure 10 a, a total of 164 sources are shown in the color-magnitude space. We identify 24 YSOs ( 7 Class I; 4 Flat-spectrum; 13 Class II) and 139 Class III sources. Additionally, one Flat-spectrum source is found in the boundary of possible contaminants and is excluded from our selected YSOs. In Figure 10a, the selected Class I, Flat-spectrum, and Class II YSOs are highlighted by red circles, red diamonds, and blue triangles, respectively.

2. Using the Spitzer 3.6, 4.5, 5.8, and $8.0 \mu \mathrm{m}$ photometric data, Gutermuth et al. (2009) provided various schemes to identify the YSOs and also various possible contaminants (e.g. broad-line active galactic nuclei (AGNs), PAH-emitting galaxies, shocked emission blobs/knots, and PAH-emission-contaminated apertures). Furthermore, the selected YSOs can be further classified into different evolutionary stages based on their slopes of the SED $\left(\alpha_{3.6-8.0}\right)$ estimated from 3.6 to $8.0 \mu \mathrm{m}$ (i.e., Class I $\left(\alpha_{3.6-8.0}>-0.3\right)$, Class II $\left(-0.3>\alpha_{3.6-8.0}>-1.6\right)$, and Class III $\left(-1.6>\alpha_{3.6-8.0}>-2.56\right)$ ) (e.g., Lada et al. 2006). More details about the YSO classifications based on the Spitzer 3.6-8.0 $\mu \mathrm{m}$ bands can be found in Dewangan and Anandarao (2011). Following the conditions given in Gutermuth et al. (2009) and Lada et al. (2006), we have also identified YSOs and various possible contaminants in our selected region around the G35.6 site. The Spitzer-GLIMPSE color-color plot ([3.6]-[4.5] vs [5.8]-[8.0]) is shown in Figure 10p. Using the Spitzer 3.6-8.0 $\mu \mathrm{m}$ photometric data, we find 28 YSOs $(8$ Class I; 20 Class II), 1 Class III, and 222 contaminants. In Figure $10 \mathrm{p}$, the selected Class I and Class II YSOs are highlighted by red circles and blue triangles, respectively.

3. Taking into account the sources having detections in the first three Spitzer-GLIMPSE bands (except 8.0 $\mu \mathrm{m}$ band), Hartmann et al. (2005) and Getman et al. (2007) use a color-color plot $([4.5]-[5.8]$ vs $[3.6]-[4.5])$ to identify the YSOs. They use color conditions, [4.5]-[5.8] $\geq 0.7$ and $[3.6]-[4.5] \geq 0.7$, to select protostars. Using the first three Spitzer-GLIMPSE bands, we obtain 4 protostars in our selected region (see Figure 10.).

4. In this YSO classification scheme, the NIR color-magnitude space $(\mathrm{H}-\mathrm{K} / \mathrm{K})$ is utilized to identify additional YSOs. We use a color $\mathrm{H}-\mathrm{K}$ value (i.e. $\sim 2.0$ ) that divides the $\mathrm{H}-\mathrm{K}$ excess sources from the rest of the population. This color condition is adopted based on the color-magnitude plot of sources from the nearby control field (size of the selected region $12^{\prime} .1 \times 8.7$; centered at: $\left.l=35^{\circ} .484 ; b=-0^{\circ} .67\right)$. Using the color $\mathrm{H}-\mathrm{K}$ cut-off condition, we find 142 embedded YSOs in the region probed in this work (see Figure $10 \mathrm{~d}$ ).

All together, the analysis of the photometric data at $1-24 \mu \mathrm{m}$ gives a total of 198 YSOs in our selected region around the G35.6 site. In Figure 11, these selected YSOs are overlaid on the integrated molecular map, helping to infer the YSOs belonging to the MCG35.6. We find 59 YSOs in the direction of EMC1, while 15 YSOs are identified toward EMC2. In the EMC1, we have found a larger number of YSOs compared to the previously reported work of Paron et al. (2011) (see Table 2 in their paper). Table 5 lists the NIR and Spitzer photometric magnitudes of the 59 YSOs seen toward EMC1, which are identified using the color-magnitude and color-color plots. The table also gives the information about the classification stages of YSOs. In EMC1, we find noticeable YSOs toward one of the edges of the semi-ring-like morphology. Furthermore, several YSOs are also distributed toward the ring-like feature in EMC1.

\subsubsection{Young stellar clusters in MCG35.6}

In this section, we probe the individual groups or clusters of YSOs based on their spatial distribution and the statistical surface density utility. The clusters of YSOs within the MCG35.6 are still unknown. In star-forming regions, the surface density map of selected YSOs is often 
generated using the nearest-neighbour (NN) technique (see Gutermuth et al. 2009, Bressert et al. 2010; Dewangan et al. 2015, 2017a; Dewangan 2017b, for more details). The surface density map can be obtained by dividing the selected field using a regular grid and extracting the surface density of YSOs at each grid point. The surface number density at the $k^{\text {th }}$ grid point is given by $\rho_{k}=(n-1) / A_{k}$ (e.g. Casertano \& Hut 1985), where $A_{k}$ denotes the surface area defined by the radial distance to the $n=6 \mathrm{NN}$. Adopting this procedure, the surface density map of all the selected 198 YSOs has been constructed using a $5^{\prime \prime}$ grid and $6 \mathrm{NN}$ at a distance of 3.7 kpc. In Figure $11 \mathrm{p}$, we show the surface density contours of YSOs overlaid on the Herschel column density map. The YSOs density contours are drawn at 3, 5, and $10 \mathrm{YSOs} / \mathrm{pc}^{2}$, increasing from the outer to the inner regions. The positions of Herschel clumps are also shown in Figure 11b. The YSO clusters are seen toward Herschel clumps (i.e., nos. 1, 2, 3, and 8). Additionally, noticeable YSOs are also found toward the Herschel clumps nos. 4 and 5 without any clustering. Earlier in Section 3.5 the five clumps (i.e., nos. 1, 2, 3, 4, and 5) have been seen at the edges of the ring-like feature (see a solid yellow box in Figure 6b). Figures $12 \mathrm{a}$ and $12 \mathrm{~b}$ also show the spatial distribution of YSOs toward the ring-like feature, depicting the ongoing star formation activities on the edges of the ring-like feature.

Star formation activity is also observed toward the clump no. 8 in EMC2. Furthermore, we find surface density contours of YSOs away from the ${ }^{13} \mathrm{CO}$ emission, implying that these clusters are not directly associated with MCG35.6.

\section{DISCUSSION}

\subsection{HII regions in EMC1}

A careful multi-wavelength data analysis reveals two distinct structures (i.e. semi-ring-like and ring-like structures) in the EMC1. The semi-ring-like structure is associated with the radio source crs1, and is possibly produced by the ionizing feedback of a B0.5V or B0V type star (see Section 3.4). On the other hand, if the ringlike feature was produced by feedback of a massive star then, there should be ionized gas inside the structure produced by a massive star located within the ring-like feature. Since there are no evidences of the existence of this star or of ionized gas (at the sensitivity of the observed NVSS radio map; $1 \sigma \sim 0.45 \mathrm{mJy} \mathrm{beam}^{-1}$ ), then the ring-like feature is unlikely to be originated by the feedback of a massive star. However, with the sensitivity limit of the NVSS data, one cannot completely rule out the presence of a massive B2-B3 star, or even a cluster of B3 stars, producing the cavity in the ring-like structure. Furthermore, the north-western side of the ring-like feature appears to be influenced by the feedback of the massive star linked with the radio source crs2 (see Section 3.4.

The photometric analysis of point-like sources reveals a population of YSOs toward the edges of the semi-ringlike and ring-like structures (see Section 3.7.1). Hence, star formation activities are traced within EMC1 (see Figure 11a and also Section 3.7). The young stellar populations can be originated spontaneously in a given molecular cloud, or the process of collapse and star for- mation can also be influenced by some external agents such as the expansion of $\mathrm{H}$ II regions (e.g., Elmegreen 1998). Hence, a triggered star formation scenario (via an expanding H II region) appears applicable in the G35.6 site. The dynamical or expansion ages of the $\mathrm{H}$ II regions are estimated to be $\sim 0.35-0.5 \mathrm{Myr}$ (for $\mathrm{n}_{0}=10^{3} \mathrm{~cm}^{-3}$; see Section 3.3, see also Paron et al. 2011), which can be treated as the indicative values or the lower limits to the actual ages. Evans et al. (2009) estimated the average lifetimes of Class 1 and Class 11 YSOs to be $\sim 0.44 \mathrm{Myr}$ and $\sim 1-3 \mathrm{Myr}$, respectively. Based on the comparison of these typical ages of YSOs and the dynamical ages of the H II regions, we cannot completely rule out the formation of Class I YSOs through the expansion of the $\mathrm{H}$ II regions in EMC1. However, the majority of selected young populations in EMC1 are Class II YSOs (see Table 551 Class II candidates out of 59 YSOs). Hence, the clusters of YSOs are unlikely to be originated by the expansion of the H II regions linked with crs1 and crs2.

\subsection{Formation and evolution of features in EMC1}

The embedded ring-like feature in EMC1 is the most prominent structure evident in the ${ }^{13} \mathrm{CO}$ integrated intensity and Herschel column density maps (see Figures 8 a and $8 \mathrm{~b}$ ). The ring-like feature with a face-on view has a central region/cavity devoid of radio continuum emission. The structure in the sky of the ring-like feature can be a spherically oblate cloud.

From a theoretical point of view, the formation of a ring-like feature can be explained by the ambipolar diffusion, when the cloud was magnetically subcritical. Conditions with low density perturbations and thermal pressure allow the cloud to condense along the field lines to form the oblate morphologies. When the cloud axis is non-axisymmetric, the magnetically mediated cloud condensation forms a ring-like feature with dense supercritical cores (Fiedler \& Mouschovias 1993). Li \& Nakamura (2002) also carried out numerical simulations to examine the evolution of subcritical clouds by considering the nonaxisymmetric case under thin-disk approximation. They reported that a magnetically subcritical cloud fragments into multiple magnetically supercritical cores, when the density perturbations are very large. Eventually, these supercritical cores lead to the birth of a new generation of stars in small clusters through gravitationally dominated ambipolar diffusion.

A detailed analysis of the GPIPS and Planck polarimetric data indicates that the magnetic field is parallel to the major axis of the ring-like feature. Our analysis on magnetic field properties further suggests that three subregions (i.e. R1, R2, and R3) in the ring-like feature are magnetically supercritical and each subregion hosts at least one Herschel clump (see Section 3.6.3). We find at least five clumps (i.e., nos. 1, 2, 3, 4, and 5; see Figure 8 a) at the edges of this ring-like feature which seem to be spatially distributed in an almost regularly spaced manner, indicating the fragmentation of the ring-like feature. A population of YSOs is found toward all the five clumps including three magnetically supercritical clumps (see Section 3.7 .2 and Figures $12 \mathrm{a}$ and $12 \mathrm{~b}$ ). One can note that the subregion R2 is seen in the direction of the north-western side of the ring-like feature, where the impact of the massive star linked with the radio source crs2 cannot be ignored (see Section 3.4). However, in the 
subregion R2, the origin of Class II YSOs through the expansion of the H II region is unlikely (see Section 4.1).

Furthermore, we have also investigated the magnetic field properties in the subregion R4 located at one of the edges of the semi-ring-like morphology (see Section 3.6. The field orientation obtained from the GPIPS H-band polarization appears perpendicular to the major axis of the molecular structure linked with the subregion R4 (see Figure 7a). As mentioned earlier, the subregion R4 is magnetically subcritical. Additionally, there is no signature of star formation activity in this particular subregion (see Section 3.7). This implies that the magnetic field might be dynamically important in the formation and evolution of the semi-ring like feature (e.g. Mouschovias 1978).

Our observational results are in agreement with the outcomes of the numerical simulations concerning the evolution of the magnetically subcritical cloud (see $\mathrm{Li}$ \& Nakamura 2002), which fragments into multiple magnetically supercritical clumps and further leads to birth of multiple stars/systems or clusters.

\section{SUMMARY AND CONCLUSIONS}

In this paper, to probe the physical processes and star formation activity, we have carried out an extensive study of the G35.6 site using multi-wavelength data. Our analysis has been focused on the distribution of the ionized gas, molecular gas, cold dust, magnetic field, and embedded young populations. The important findings of this work are:

- The molecular cloud associated with the G35.6 site (i.e. MCG35.6) is depicted in the velocity range 53-62 $\mathrm{km} \mathrm{s}^{-1}$. In our selected region around the G35.6 site, two extended molecular condensations (i.e., EMC1 and EMC2) are observed.

- The molecular data trace two molecular components in the direction of the G35.6 site. A molecular component associated with EMC1 is depicted in the velocity range 53-59 $\mathrm{km} \mathrm{s}^{-1}$, while the other molecular component associated with EMC2 is observed at $59-62 \mathrm{~km} \mathrm{~s}^{-1}$. A careful analysis of the molecular line data shows that these two molecular components are clearly separated in both space and velocity, and seem to be unrelated molecular clouds.

- In EMC1, the multi-wavelength images reveal the existence of a semi-ring-like feature (associated with the ionized gas emission) and an embedded face-on ring-like feature devoid of radio continuum emission in its center. The detection/non-detection of the ionized gas emission in the G35.6 site is obtained from the NVSS radio continuum map at $1.4 \mathrm{GHz}$, which has the sensitivity limit of $\sim 0.45 \mathrm{mJy}$ beam $^{-1}$.

- Two compact radio continuum sources (i.e., crs1 and crs2) are associated with EMC1. The NVSS $1.4 \mathrm{GHz}$ continuum data analysis reveals that each $\mathrm{H}$ II region is excited by a B0.5V-B0V type star and has a dynamical age of $\sim 0.35-0.5 \mathrm{Myr}$.

- The Herschel temperature map reveals the heating from the two compact radio continuum sources with temperatures $\sim 22-27 \mathrm{~K}$, while the ring-like feature has a temperature range of about $18-20 \mathrm{~K}$.

- In the Herschel column density map, at least five massive Herschel clumps $\left(M_{\text {clump }} \sim 740-1420 \mathrm{M}_{\odot}\right)$ seem to be spatially distributed in an almost regularly spaced manner along the ring-like feature.

- The analysis of photometric data at 1-24 $\mu \mathrm{m}$ reveals a total of 198 YSOs. Most of these YSOs are spatially distributed toward the Herschel clumps in EMC1.

- Star formation activities have been found toward the clumps seen at the edges of the ring-like feature in EMC1.

- The Planck and GPIPS polarimetric data trace the POS magnetic field parallel to the major axis of the ring-like feature.

- Using the GPIPS H-band polarimetric data, three magnetically supercritical subregions containing the Herschel clumps are observed in the ring-like feature.

Considering our observational findings, the semi-ringlike feature is resultant of the ionizing feedback of a massive star linked with the radio source crs1. On the other hand, the existence of a ring-like feature cannot be explained by the impact of massive star(s). Our observational analysis favors the idea that the ring-like feature may have formed from a magnetically dominated cloud. The results derived using the molecular emission, cold dust emission, and magnetic field properties reveal that the ring-like feature contains multiple supercritical clumps that can potentially fragment into dense cores, eventually leading to the birth of multiple stars/systems or clusters. These findings can be linked to the formation and evolution of the cloud via the magnetic field mediated mechanism as suggested by Li \& Nakamura (2002).

We thank the anonymous reviewer for a critical reading of the manuscript and several useful comments and suggestions, which greatly improved the scientific contents of the paper. The research work at Physical Research Laboratory is funded by the Department of Space, Government of India. This work is based on data obtained as part of the UKIRT Infrared Deep Sky Survey. This publication made use of data products from the Two Micron All Sky Survey (a joint project of the University of Massachusetts and the Infrared Processing and Analysis Center / California Institute of Technology, funded by NASA and NSF), archival data obtained with the Spitzer Space Telescope (operated by the Jet Propulsion Laboratory, California Institute of Technology under a contract with NASA). This publication makes use of molecular line data from the Boston University-FCRAO Galactic Ring Survey (GRS). The GRS is a joint project of Boston University and Five College Radio Astronomy Observatory, funded by the National Science Foundation (NSF) under grants AST-9800334, AST-0098562, and AST-0100793. This publication makes use of the Galactic Plane Infrared Polarization Survey (GPIPS). The GPIPS was conducted using the Mimir instrument, jointly developed at Boston University and Lowell Observatory and supported by NASA, NSF, and the W.M. Keck Foundation. $\mathrm{RD}$ acknowledges CONACyT(México) for the PhD grant 370405 .

\section{REFERENCES}

Anderson, L. D., Bania, T. M., Jackson, J. M., et al. 2009, ApJS, 181,255

Andersson, B. G., Lazarian, A., Vaillancourt, J. E. 2015,

ARA\&A, 53, 501 

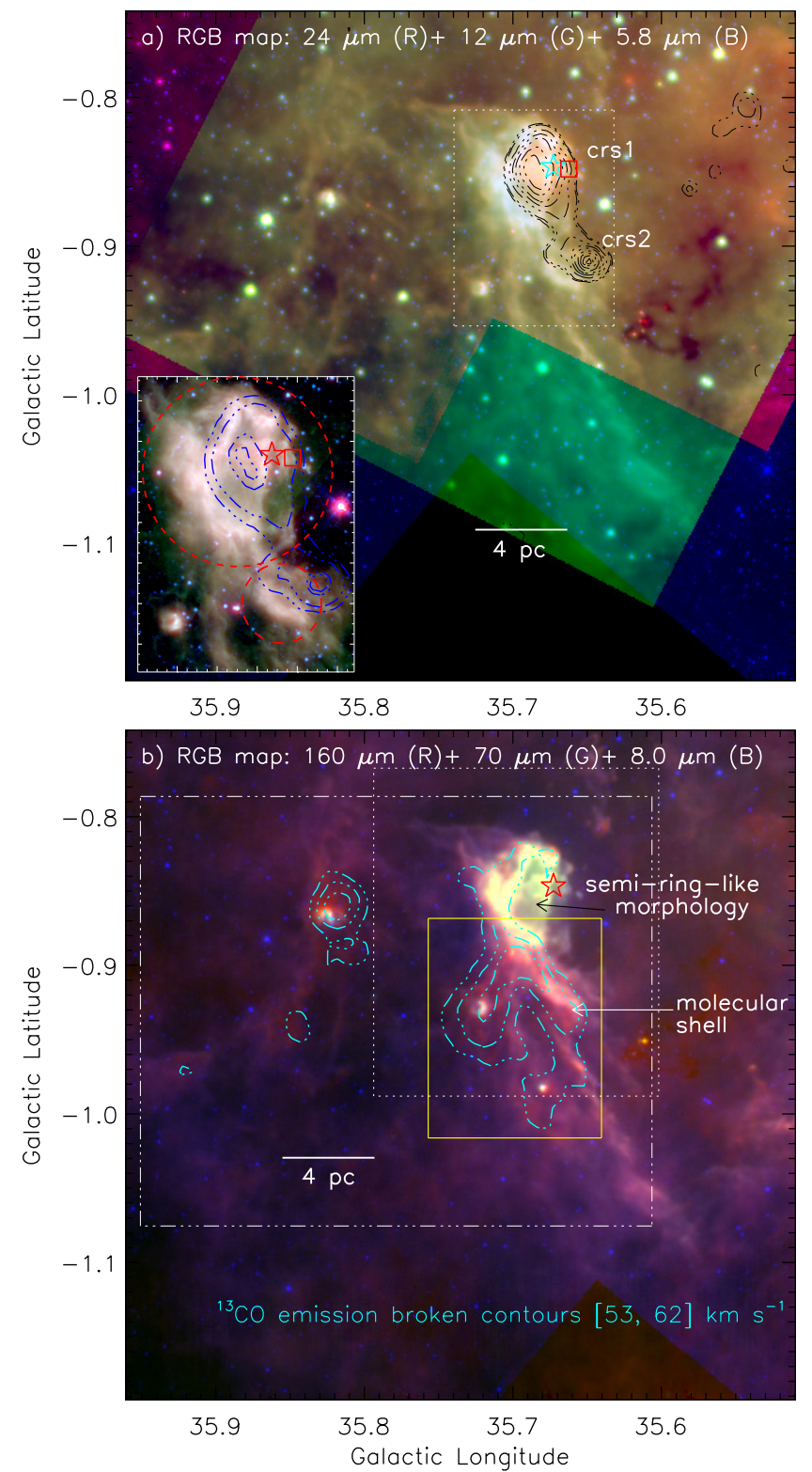

Figure 1. A large-scale view of the G35.6 site (size of the selected field $\sim 27^{\prime} \times 27^{\prime}$; central coordinates: $l=35^{\circ} .735 ; b=-0^{\circ} .967$ ). a) The image is the result of the combination of three bands (in log scale): $24.0 \mu \mathrm{m}$ in red (Spitzer), $12.0 \mu \mathrm{m}$ in green (WISE), and $5.8 \mu \mathrm{m}$ in blue (Spitzer). Contours of NVSS $1.4 \mathrm{GHz}$ radio continuum emission (beam size $\sim 45^{\prime \prime}$ ) are superimposed with $8,10,20,30,40,55,70,85$, and $95 \%$ of the peak value (i.e., $31.7 \mathrm{mJy}$ /beam). Two compact radio sources (i.e., crs1 and crs2) traced in the NVSS $1.4 \mathrm{GHz}$ map are highlighted in the figure. The inset on the bottom left shows the $\mathrm{H}$ II regions in zoomed-in view, using a color-composite image (WISE $12.0 \mu \mathrm{m}$ (in red), Spitzer $8.0 \mu \mathrm{m}$ (in green), and Spitzer $5.8 \mu \mathrm{m}$ (in blue)) overlaid with the NVSS radio continuum emission at $1.4 \mathrm{GHz}$ (see a dashed white box in figure). In the inset, the NVSS contours are $2.5,6,20$, and $25 \mathrm{mJy} / \mathrm{beam}$. A red square indicates the position of a CORNISH $5 \mathrm{GHz}$ radio source, G035.6624-00.8481 (see also the inset). b) The image is the result of the combination of three bands: Herschel $160 \mu \mathrm{m}$ (red), Herschel $70 \mu \mathrm{m}$ (green), and Spitzer $8.0 \mu \mathrm{m}$ (blue). The composite map is also overlaid with the ${ }^{13} \mathrm{CO}$ emission contours integrated over a velocity interval from 53 to $62 \mathrm{~km} \mathrm{~s} \mathrm{~s}^{-1}$. The ${ }^{13} \mathrm{CO}$ contours are shown with the levels of 4.5, 8 , and $12 \mathrm{~K} \mathrm{~km} \mathrm{~s}^{-1}$. A small dotted box (in white) shows the area investigated by Paron et al. (2011). A semi-ring-like morphology and a molecular shell are also highlighted in the figure (see also Paron et al. 2011). A solid box (in yellow) encompasses the area shown in Figure 2 A big dotted-dashed box (in white) encompasses the area shown in Figures 3 and 4 a. In all the panels, a star symbol indicates the position of IRAS 18569+0159. The scale bar corresponding to $4 \mathrm{pc}$ (at a distance of $3.7 \mathrm{kpc}$ ) is shown in both the panels.

Arce, H. G., Borkin, M. A., Goodman, A. A., Pineda, J. E.,\& Beaumont, C. N. 2011, ApJ, 742, 105

Beaumont, C. N., \& Williams, J. P. 2010, ApJ, 709, 791

Benjamin, R. A.,Churchwell, E., Babler, B. L., et al. 2003, PASP, 115,953

Bisbas, T. G., Wünsch, R., Whitworth, A. P., \& Hubber, D. A. 2009, A\&A, 497, 649

Bohlin, R. C., Savage, B. D., \& Drake, J. F. 1978, ApJ, 224, 13233

Bressert, E., Bastian, N., Gutermuth, R., et al. 2010, MNRAS, 409, 54

Bressert, E., Ginsburg, A., Bally, J., et al. 2012, ApJ, 758, 28
Carey, S. J., Noriega-Crespo, A., Price, S. D., et al. 2005, BAAS, 37,1252

Casertano, S., \& Hut P. 1985, ApJ, 298, 80

Chandrasekhar, S., \& Fermi, E. 1953, ApJ, 118, 113

Chen, Z., Jiang, Z., Tamura, M., Kwon, J., \& Roman-Lopes, A. 2017, ApJ, 838, 80

Churchwell, E., Povich, M. S., Allen, D., et al. 2006, ApJ, 649, 759

Churchwell, E., Watson, D. F., Povich, M. S., et al. 2007, ApJ, 670,428

Clemens, D. P., Pavel, M. D., \& Cashman, L. R. 2012a, ApJS, 200, 21 


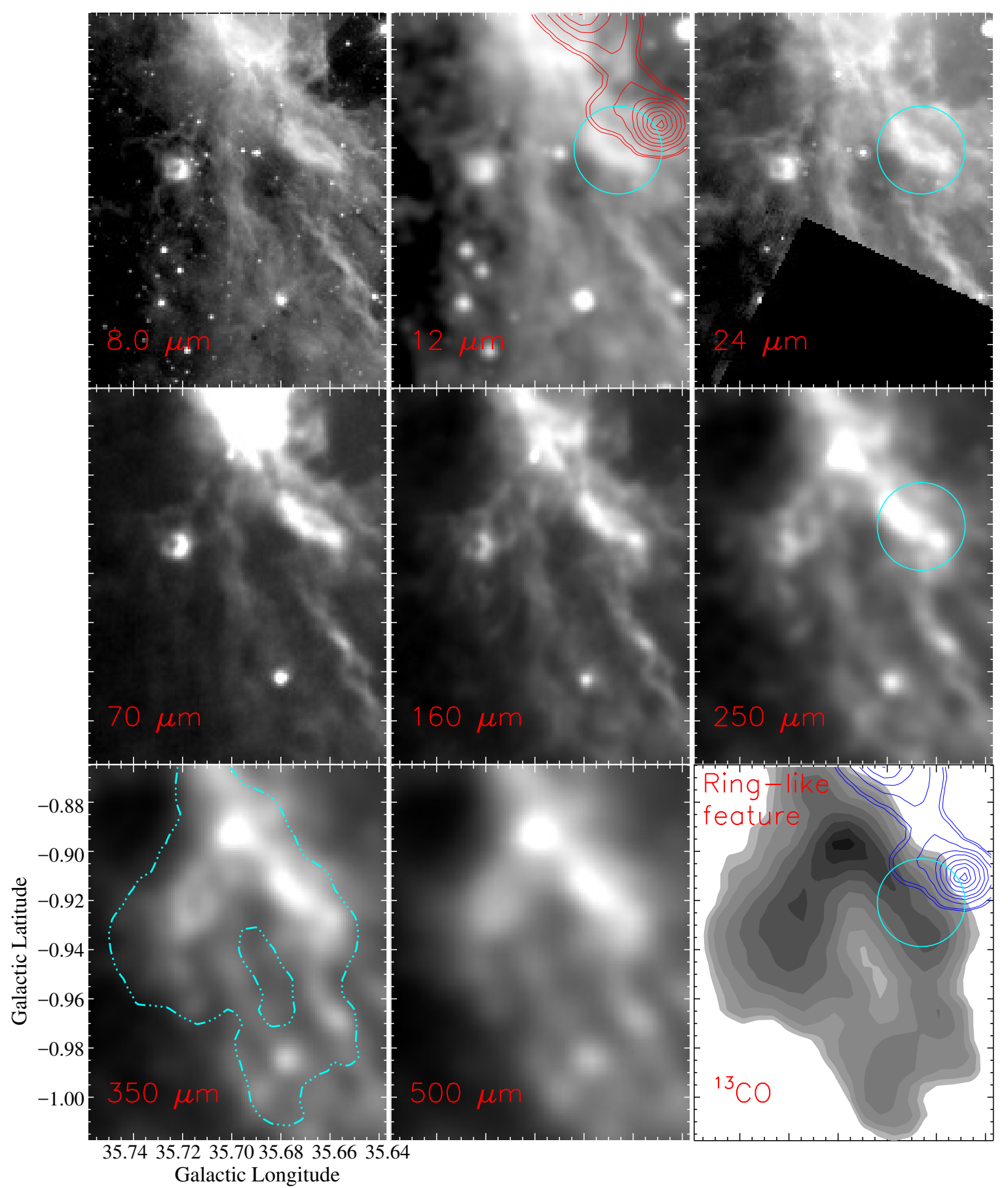

Figure 2. A zoomed-in multi-wavelength view of a ring-like feature. The panels present images at $8.0 \mu \mathrm{m}, 12 \mu \mathrm{m}, 24 \mu \mathrm{m}, 70 \mu \mathrm{m}, 160 \mu \mathrm{m}, 250$ $\mu \mathrm{m}, 350 \mu \mathrm{m}$, and $500 \mu \mathrm{m}$, and integrated ${ }^{13} \mathrm{CO}$ contour map, from the GLIMPSE, WISE, MIPSGAL, Hi-GAL, and GRS surveys (from left to right in increasing order). The integrated ${ }^{13} \mathrm{CO}$ contour map is similar to the one shown in Figure $5 \mathrm{k}$. The integrated ${ }^{13} \mathrm{CO}$ contour map and the image at $12 \mu \mathrm{m}$ are overlaid with the NVSS $1.4 \mathrm{GHz}$ continuum emission contours, which are similar to the one shown in Figure $1 \mathrm{p}$. A ${ }^{13} \mathrm{CO}$ contour is superimposed on the $350 \mu \mathrm{m}$ map with a level of $3.8 \mathrm{~K} \mathrm{~km} \mathrm{~s}^{-1}$. The ring-like feature is prominently evident at wavelengths longer than $160 \mu \mathrm{m}$, and does not contain ionized gas emission at its interior. A small feature seen in the infrared and sub-mm images is also highlighted by a circle in four panels. 


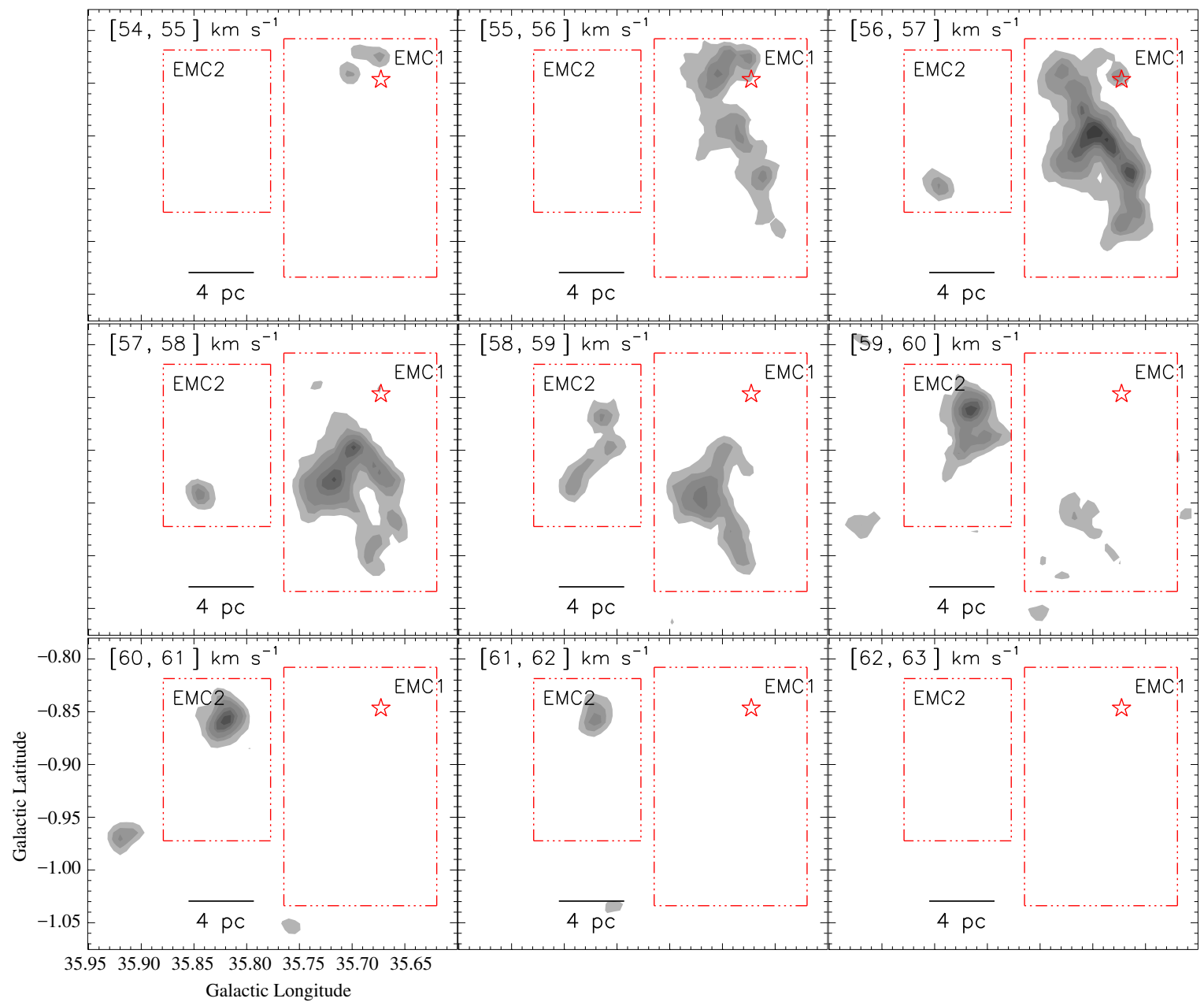

Figure 3. The ${ }^{13} \mathrm{CO}(\mathrm{J}=1-0)$ velocity channel contour maps in the direction of the site G35.6 (size of the selected field $\sim 21^{\prime} \times 17^{\prime} .7$; central coordinates: $l=35^{\circ} .778 ; b=-0^{\circ} .931$ ). The molecular emission is integrated over a velocity interval, which is given in each panel (in $\mathrm{km} \mathrm{s}^{-1}$ ). The contours are shown with the levels of $1,2,3,5,6,7$, and $8.5 \mathrm{~K} \mathrm{~km} \mathrm{~s}^{-1}$. Two extended molecular condensations (i.e., EMC1 and EMC2) are labeled in the maps. The fields of EMC1 and EMC2 are also shown by dotted-dashed boxes in each map. In each panel, a star symbol indicates the position of IRAS $18569+0159$. The map at $[57,58] \mathrm{km} \mathrm{s}^{-1}$ traces an almost ring-like feature.

Table 1

List of surveys (covering from NIR to radio wavelengths) adopted in this work.

\begin{tabular}{|c|c|c|c|}
\hline Survey & Wavelength $(\mathrm{s})$ & Resolution $\left({ }^{\prime \prime}\right)$ & Reference \\
\hline Two Micron All Sky Survey (2MASS) & $1.25-2.2 \mu \mathrm{m}$ & $\sim 2.5$ & Skrutskie et al. 2006 \\
\hline Galactic Plane Infrared Polarization Survey (GPIPS) & $1.6 \mu \mathrm{m}$ & $\sim 1.5$ & Clemens et al. (2012a) \\
\hline UKIRT NIR Galactic Plane Survey (GPS) & $1.25-2.2 \mu \mathrm{m}$ & $\sim 0.8$ & Lawrence et al. 2007 \\
\hline Spitzer Galactic Legacy Infrared Mid-Plane Survey Extraordinaire (GLIMPSE) & $3.6,4.5,5.8,8.0 \mu \mathrm{m}$ & $\sim 2, \sim 2, \sim 2, \sim 2$ & Benjamin et al. 2003 \\
\hline Wide Field Infrared Survey Explorer (WISE) & $3.4,4.6,12,22 \mu \mathrm{m}$ & $\sim 6, \sim 6.5, \sim 6.5, \sim 12$ & Wright et al. 2010 \\
\hline Spitzer MIPS Inner Galactic Plane Survey (MIPSGAL) & $24 \mu \mathrm{m}$ & $\sim 6$ & Carev et al. 2005 \\
\hline Herschel Infrared Galactic Plane Survey (Hi-GAL) & $70,160,250,350,500 \mu \mathrm{m}$ & $\sim 5.8, \sim 12, \sim 18, \sim 25, \sim 37$ & Molinari et al. 2010 \\
\hline Planck polarization data & $850 \mu \mathrm{m}$ & $\sim 294$ & Planck Collaboration IX 2014 \\
\hline Galactic Ring Survey (GRS) & $2.7 \mathrm{~mm} ;{ }^{13} \mathrm{CO}(\mathrm{J}=1-0)$ & $\sim 45$ & Jackson et al. $(2006)$ \\
\hline Coordinated Radio and Infrared Survey for High-Mass Star Formation (CORNISH) & $6 \mathrm{~cm}$ & $\sim 1.5$ & Hoare et al. 2012 \\
\hline NRAO VLA Sky Survey (NVSS) & $21 \mathrm{~cm}$ & $\sim 45$ & Condon et al. 1998 \\
\hline
\end{tabular}



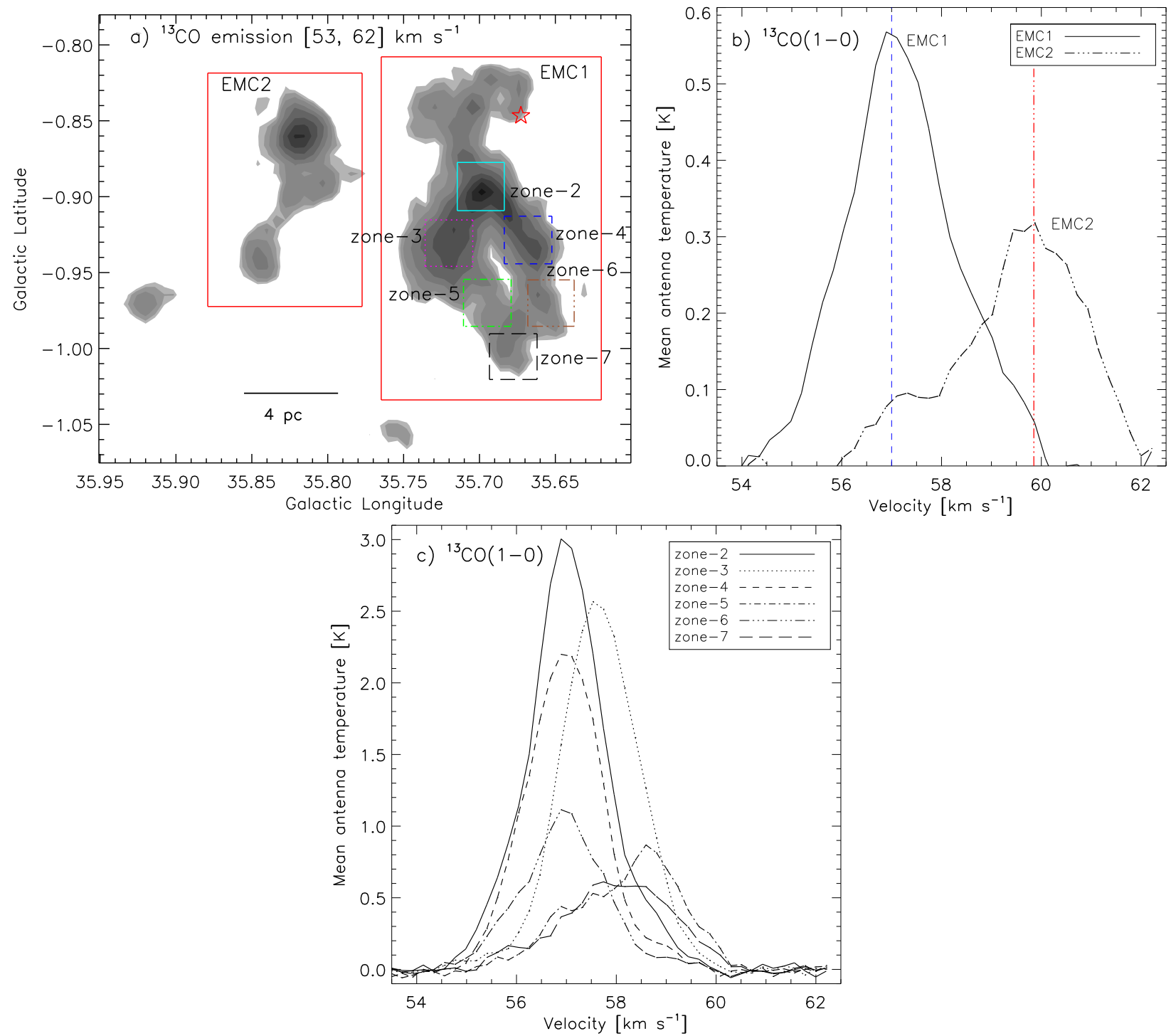

Figure 4. a) Molecular emission contour map integrated over a velocity interval from 53 to $62 \mathrm{~km} \mathrm{~s}^{-1}$. The ${ }^{13} \mathrm{CO}$ contours are $19.034 \mathrm{~K} \mathrm{~km}$ $\mathrm{s}^{-1} \times(0.12,0.15,0.2,0.3,0.4,0.55,0.7,0.85,0.95)$. Two extended molecular condensations (i.e., EMC1 and EMC2) are labeled in the map. In the molecular map, the areas of several fields (i.e. EMC1, EMC2, zone-2, zone-3, zone-4, zone-5, zone-6, and zone-7) are also highlighted by boxes. b) The GRS ${ }^{13} \mathrm{CO}(1-0)$ profile in the direction of two fields, EMC1 and EMC2 (see corresponding box in Figure 4 ). Two velocity peaks are marked by broken lines. c) The GRS ${ }^{13} \mathrm{CO}(1-0)$ spectra in the direction of six small fields linked with the ring-like feature (i.e. zone-2 to zone-7; see corresponding boxes in Figure $4 \mathrm{k}$ ). In the last two panels, each spectrum is obtained by averaging the area highlighted by a box.

Table 2

Physical properties of the Herschel clumps in our selected star-forming region (see Figures 6 p and 6 ). Column 1 gives the IDs assigned to the clumps. Table also lists positions, deconvolved effective radius $\left(\mathrm{R}_{c}\right)$, clump mass $\left(\bar{M}_{\text {clump }}\right)$, peak column density $\left(\mathrm{N}\left(\mathrm{H}_{2}\right)\right)$, and extinction $\left(A_{V}=1.07 \times 10^{-21} N\left(\mathrm{H}_{2}\right)\right)$ (Bohlin et al. 1978). The clumps highlighted with superscript daggers are found at the edges of the ring-like feature (see also Figures 8 and 12 .

\begin{tabular}{ccccccr}
\hline ID & $\begin{array}{c}l \\
(\text { degree })\end{array}$ & $\begin{array}{c}b \\
(\text { degree })\end{array}$ & $\begin{array}{c}\mathrm{R}_{c} \\
(\mathrm{pc})\end{array}$ & $\begin{array}{c}M_{\text {clump }} \\
\left(M_{\odot}\right)\end{array}$ & $\begin{array}{c}\text { peak }\left(\mathrm{H}_{2}\right) \\
\left(10^{21} \mathrm{~cm}^{-2}\right)\end{array}$ & $\begin{array}{r}\mathrm{A}_{V} \\
(\mathrm{mag})\end{array}$ \\
\hline \hline $1^{\dagger}$ & 35.702 & -0.899 & 1.7 & 1150 & 10 & 11 \\
$2^{\dagger}$ & 35.726 & -0.954 & 1.5 & 740 & 5.4 & 6 \\
$3^{\dagger}$ & 35.683 & -0.919 & 1.9 & 1420 & 8.5 & 9 \\
$4^{\dagger}$ & 35.679 & -0.993 & 1.9 & 1275 & 7.7 & 8 \\
$5^{\dagger}$ & 35.664 & -0.973 & 1.7 & 950 & 6.6 & 7 \\
6 & 35.854 & -0.946 & 1.5 & 715 & 5.9 & 6 \\
7 & 35.881 & -0.927 & 1.2 & 450 & 5.6 & 6 \\
8 & 35.831 & -0.864 & 3.2 & 3390 & 9.1 & 10 \\
9 & 35.897 & -0.911 & 0.8 & 175 & 4.2 & 5 \\
\hline
\end{tabular}



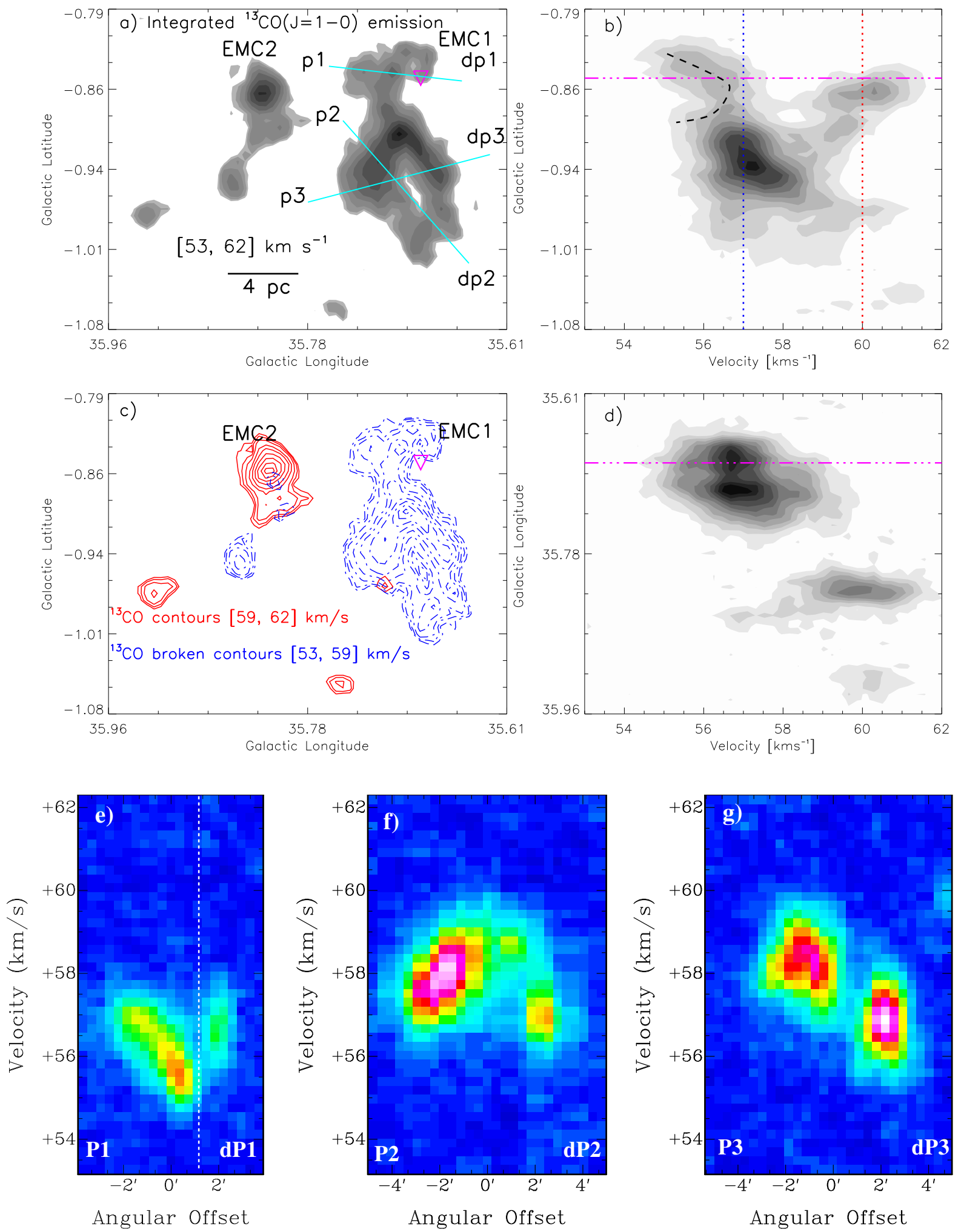

Figure 5. a) A contour map of integrated ${ }^{13} \mathrm{CO}$ emission in the direction of the Galactic $\mathrm{H}$ II region G35.6. The ${ }^{13} \mathrm{CO}$ integrated velocity range is from $53 \mathrm{~km} \mathrm{~s}^{-1}$ to $62 \mathrm{~km} \mathrm{~s}^{-1}$. The ${ }^{13} \mathrm{CO}$ contours are similar to those shown in Figure $4 \mathrm{k}$. Each solid line (in cyan) represents the axis, where the position-velocity diagrams are extracted and are shown in Figures 5 , , 5, and 5 ;. b) Latitude-velocity distribution of ${ }^{13} \mathrm{CO}$. The ${ }^{13} \mathrm{CO}$ emission is integrated over the longitude from $35^{\circ} .61$ to $35^{\circ} .96$. There are two velocity peaks (at $\sim 57 \mathrm{~km} \mathrm{~s}^{-1}$ and $\sim 60 \mathrm{~km} \mathrm{~s}{ }^{-1}$ ) seen in the position-velocity diagram which are highlighted by dotted lines (in red and blue). An inverted C-like feature is also marked by a dashed curve (in black) (see also the text). c) Two molecular components in the direction of the G35.6 site. Red contours are the ${ }^{13} \mathrm{CO}$ emission from 59 to $62 \mathrm{~km} \mathrm{~s}{ }^{-1}$ with levels of $15.219 \mathrm{~K} \mathrm{~km} \mathrm{~s}^{-1} \times(0.12,0.15,0.2,0.3,0.4,0.55,0.7,0.85$, and 0.95$)$. Blue contours (dotted-dashed) are the ${ }^{13} \mathrm{CO}$ emission from 53 to $59 \mathrm{~km} \mathrm{~s}{ }^{-1}$ with levels of $18.787 \mathrm{~K} \mathrm{~km} \mathrm{~s}^{-1} \times(0.12,0.15,0.2,0.3,0.4,0.55,0.7,0.85$, and 0.95$)$. d) Longitude-velocity distribution of ${ }^{13} \mathrm{CO}$. The ${ }^{13} \mathrm{CO}$ emission is integrated over the latitude from $-0 .{ }^{\circ} 79$ to $-1 .^{\circ} 08$. In the first two top left panels, an upside down triangle symbol indicates the position of crs1 and two extended molecular condensations (i.e., EMC1 and EMC2) are labeled. In the first two top right panels, a dotted-dashed line shows the position of crs1. e) A position-velocity diagram along the axis "p1-dp1" as shown in Figure 5 3 . A dotted line indicates the position of crs1. f) A position-velocity diagram along the axis "p2-dp2" as shown in Figure 57. g) A position-velocity diagram along the axis "p3-dp3" as shown in Figure 5 . 

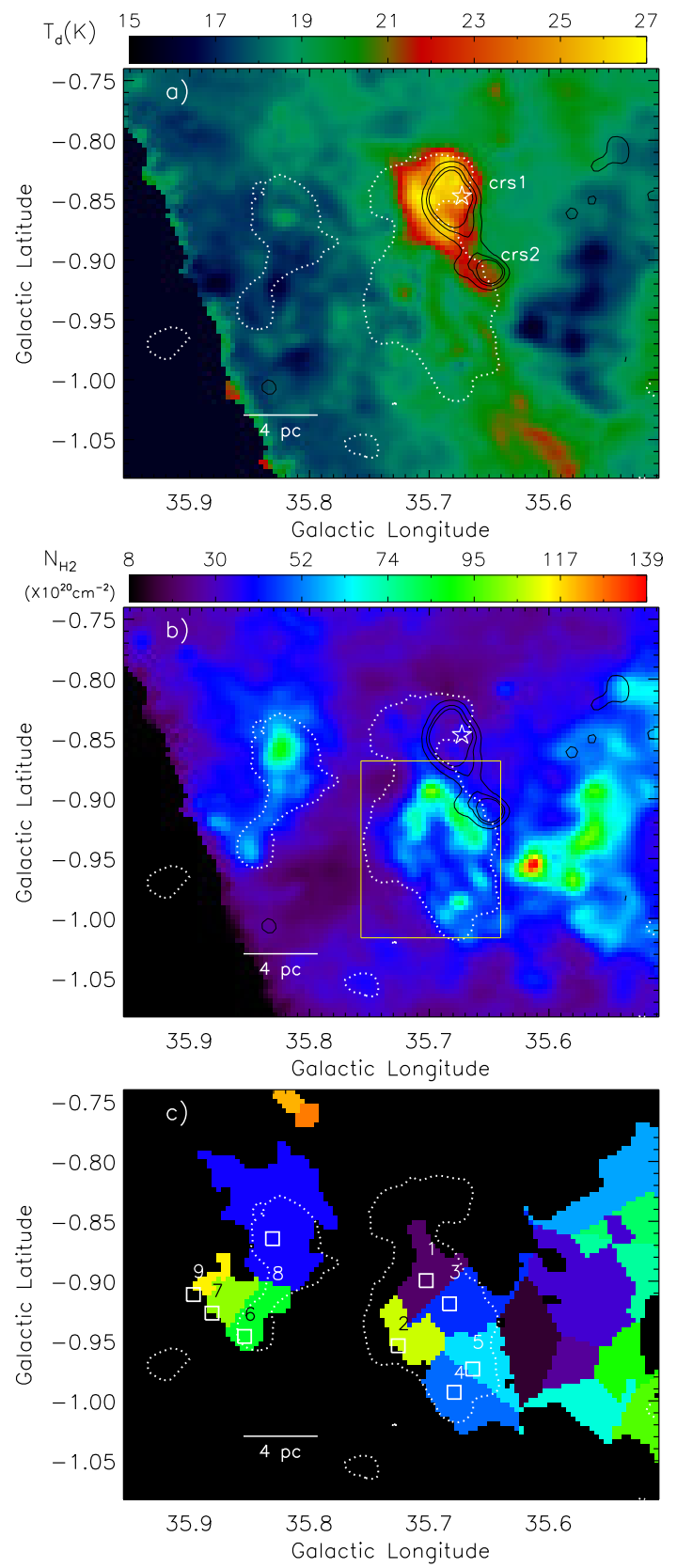

Figure 6. a) Herschel temperature map of the Galactic H II region G35.6 (size of the selected field $\sim 27^{\prime} \times 20^{\prime} 5$; central coordinates: $l=35^{\circ} .734$; $\left.b=-0^{\circ} .914\right)$. b) Herschel column density $\left(N\left(\mathrm{H}_{2}\right)\right)$ map of the Galactic $\mathrm{H}$ II region G35.6. The extinction can also be inferred using the relation $A_{V}=1.07 \times 10^{-21} N\left(\mathrm{H}_{2}\right)$ (Bohlin et al. 1978). c) The boundary of each identified clump is highlighted along with its corresponding clump ID and position (see Table 21. The ring-like feature is also observed in the Herschel column density map (see a solid yellow box in Figure 6a). In the top two panels, a star symbol indicates the position of IRAS $18569+0159$ and contours of NVSS 1.4 GHz continuum emission (solid black) are superimposed with 8,10 , and $60 \%$ of the peak value (i.e., $31.7 \mathrm{mJy} /$ beam). In all the panels, the ${ }^{13} \mathrm{CO}$ emission contour (dotted white) is also shown from 53 to $62 \mathrm{~km} \mathrm{~s}^{-1}$ with a level of $2.15 \mathrm{~K} \mathrm{~km} \mathrm{~s}^{-1}$. In each panel, the scale bar at the bottom-left corner corresponds to $4 \mathrm{pc}$ (at a distance of $3.7 \mathrm{kpc})$.

Clemens, D. P., Pinnick, A., Pavel, M. D. et. al 2012b, ApJS, 200, 19

Clemens, D. P., Tassis, K., \& Goldsmith, P. F. 2016, ApJ, 833, 176

Condon, J. J., Cotton, W. D., Greisen, E. W., et al. 1998, AJ, 115,1693

Crutcher, R. M., Nutter, D. J., Ward-Thompson, D., et al. 2004, ApJ, 600, 279

Davis, L., Jr., \& Greenstein, J. L. 1951, ApJ, 114, 206

de Graauw, T., Helmich, F. P., Phillips, T. G., et al. 2010, A\&A, 518, L4

Deharveng, L., Schuller, F., Anderson, L. D., et al. 2010, A\&A, 523,6
Dewangan, L. K., \& Anandarao, B. G 2011, MNRAS, 414, 1526 Dewangan, L. K., Luna, A., Ojha, D. K., et al. 2015, ApJ, 811, 79 Dewangan, L. K., Ojha, D. K., Luna, A., et al. 2016, ApJ, 819, 66 Dewangan, L. K., Ojha, D. K., Zinchenko, I., Janardhan, P., \& Luna, A. 2017a, ApJ, 834, 22

Dewangan, L. K. 2017b, accepted in ApJ, arXiv:1702.00007

Dolginov, A. Z., \& Mitrofanov, I. G. 1976, Ap\&SS, 43, 291

Dyson, J. E., \& Williams, D. A. 1980, Physics of the Interstellar Medium (New York: Halsted Press)

Elmegreen, B. G. 1998, in ASP Conf. Ser. 148, Origins, ed. C. E. Woodward, J. M. Shull, \& H. A. Thronson, Jr. (San Francisco, CA: ASP), 150 


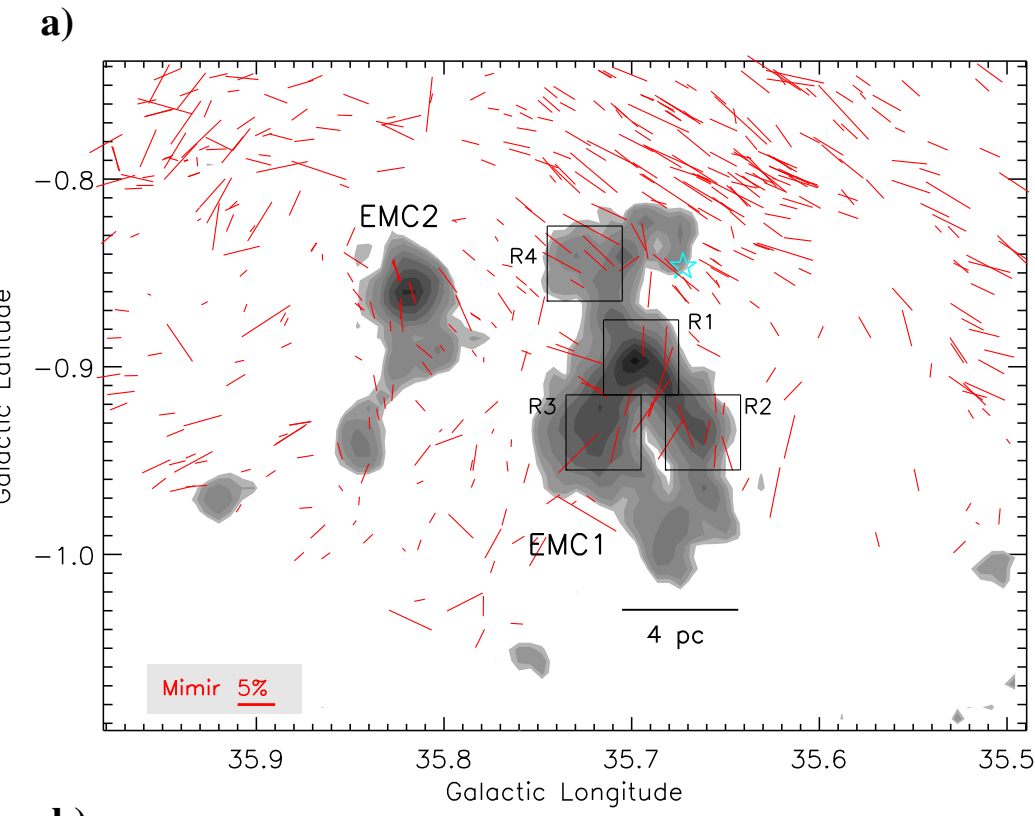

b)

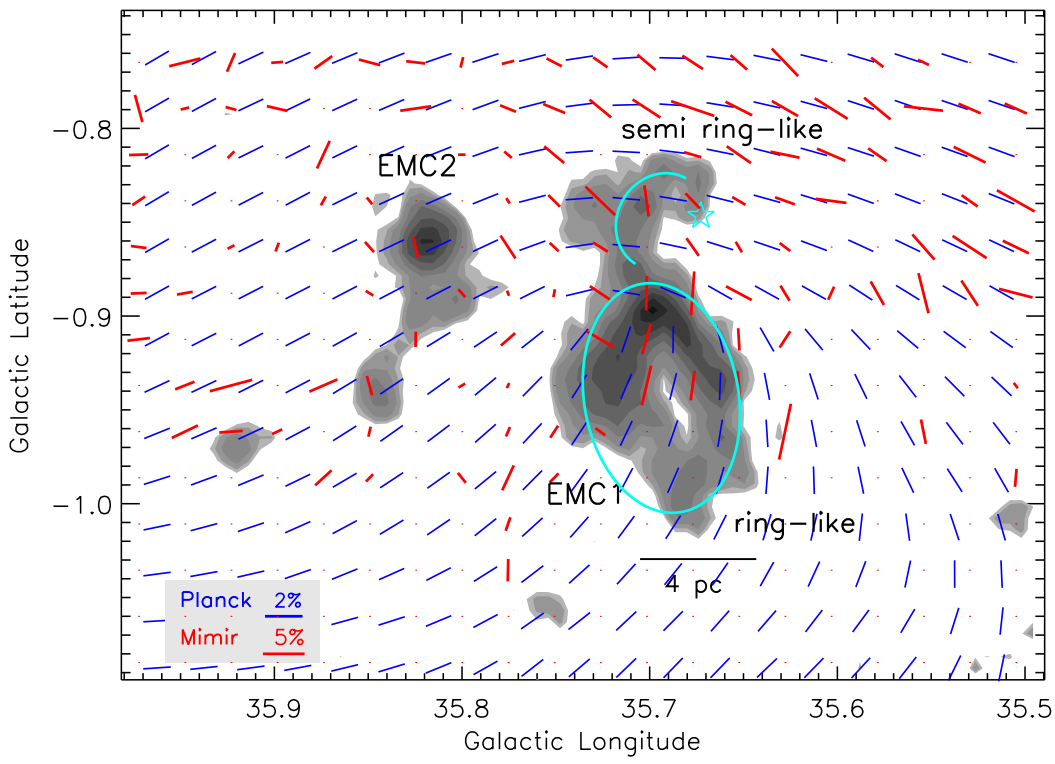

Figure 7. The polarization measurements toward the MCG35.6. a) The GPIPS H-band polarization vectors (in red color) of high quality background stars are drawn on the molecular intensity map. These stars are selected using the criteria $P / \sigma_{P}>2, \sigma_{P} \leq 5, H<13$ and $J-H>1$. The four subregions targeting the dense clumps/cores are marked by square boxes. The length of each vector shows the degree of polarization. The orientation of each vector shows the Galactic position angle of polarization. b) The Planck polarization vectors (in blue) from the dust emission at $353 \mathrm{GHz}$ are overlaid on the molecular intensity map. The vectors are rotated by $90^{\circ}$ to infer the POS magnetic field directions. The mean GPIPS polarization vectors (in red) are also overplotted on the molecular map and are obtained by spatially averaging the values matching the same pixel scale of the Planck data. In both the panels, the background map is similar to the one shown in Figure 5 . In each panel, a reference vector in the lower left corner is also shown for comparison.

Evans, N. J., II, Dunham, M. M., Jørgensen, J. K., et al. 2009, ApJS, 181, 321

Flaherty, K. M., Pipher, J. L., Megeath, S. T., et al. 2007, ApJ, 663,1069

Fiedler, R., \& Mouschovias, T. 1993, ApJ, 415, 680

Getman, K. V., Feigelson, E. D., Garmire,G., Broos, P., \& Wang, J. 2007, ApJ, 654, 316

Goicoechea, J. R., Cuadrado, S., Pety, J., et al. 2017, A\&A, 601L, 9

Górski, K. M., Hivon, E., Banday, A. J., et al. 2005, ApJ, 622, 759 Griffin, M. J., Abergel, A., Abreu, A, et al. 2010, A\&A, 518L, 3

Guieu, S., Rebull, L. M., Stauffer, J. R., et al. 2010, ApJ, 720, 46 Gutermuth, R. A., Megeath, S. T., Myers, P. C., et al. 2009, ApJS, 184, 18

Gutermuth, R. A., \& Heyer,M. 2015, AJ, 149, 64
Hamaker, J. P. \& Bregman, J. D. 1996, A\&AS, 117, 161

Hartmann, L., Ballesteros-Paredes, J., \& Bergin, E. A. 2001, ApJ, 562,852

Hartmann, L., Megeath, S. T., Allen, L., et al. 2005, ApJ, 629, 881

Hildebrand, R. H. 1983, QJRAS, 24, 267

Hoare, M. G., Purcell, C. R., Churchwell, E. B., et al. 2012, PASP, 124, 939

Hollenbach, D. J. \& Tielens, A. G. G. M. 1997, ARA\&A, 35, 179 Jackson, J. M., Rathborne, J. M., Shah, R. Y., et al. 2006, ApJS, 163,145

Kauffmann, J., Bertoldi, F., Bourke, T. L., Evans, II, N. J.,\& Lee, C. W. 2008, ApJ, 487, 993

Kendrew, S., Simpson, R., Bressert, E., et al. 2012, ApJ, 755, 71 Kurtz, S. 2005, IAUS, 227, 111 

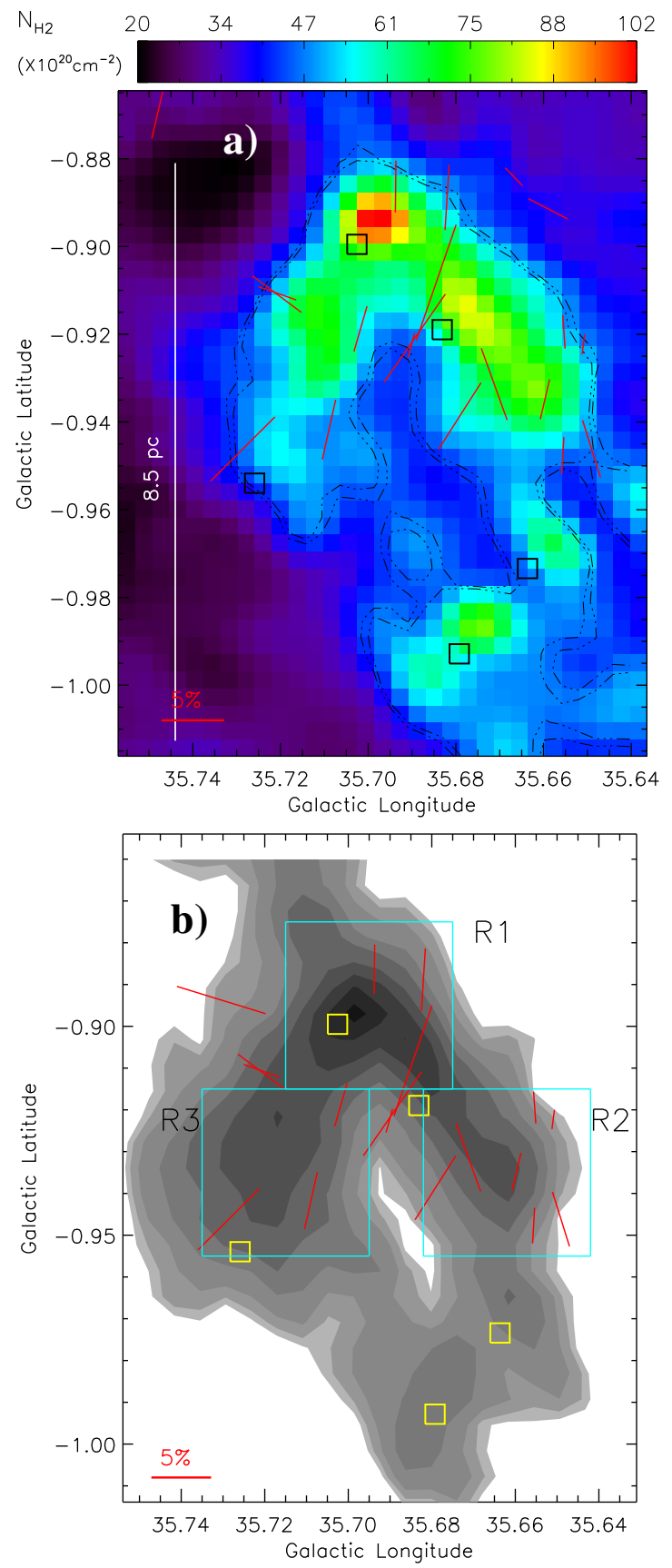

Figure 8. The H-band polarimetric measurements toward the ring-like feature (see Table 3). a) Overlay of GPIPS H-band polarization vectors (in red color) of high quality background stars on the Herschel column density map. Column density contours are also shown with levels of [4.5, 4.67] $\times 10^{21} \mathrm{~cm}^{-2}$. b) Overlay of GPIPS H-band polarization vectors (in red color) of high quality background stars on the integrated ${ }^{13} \mathrm{CO}$ emission map. Three subregions (i.e. R1, R2, and R3) targeting the areas of high column density are marked in big square boxes. A reference vector is also shown in each panel. In both the panels, the Herschel clumps are also marked by small squares. At least five clumps appear to be distributed in an almost regularly spaced manner along the ring-like feature.

Kwan, J. 1997, ApJ, 489, 284

Lada, C. J., Huard, T. L., Crews, L. J., \& Alves, J. F. 2004, ApJ, 610,303

Lada, C. J., Muench, A. A., Luhman, K. L., et al. 2006, AJ, 131, 1574

Lazarian, A. 2007, J. Quant. Spec. Radiat. Transf. 106, 225

Lawrence, A., Warren, S. J., Almaini, O., et al. 2007, MNRAS, 379,1599

Li, Z. Y. \& Nakamura, F. 2002, ApJ, 578, 256

Lockman, F. J. 1989, ApJS, 71, 469

Lucas, P. W., Hoare, M. G., Longmore, A., et al. 2008, MNRAS, 391,1281
Mallick, K. K., Ojha, D. K., Tamura, M., et al. 2015, MNRAS, 447, 2307

Martins, F., Schaerer, D., \& Hillier, D. J. 2005, A\&A, 436, 1049

Matsakis, D. N., Evans, N. J., II, Sato, T., \& Zuckerman, B. 1976, AJ, 81, 172

Molinari, S., Swinyard, B., Bally, J., et al., 2010, A\&A, 518, L100 Mouschovias, T. C. 1978, in IAU Colloq. 52: Protostars and Planets, ed. T. Gehrels, 209-242

Nakamura, F. \& Li, Z. Y. 2002, ApJ, 566, 101

Olnon, F. M. 1975, A\&A, 39, 217

Oskinova, L. M., Todt, H., Ignace, R., et al. 2011, MNRAS, 416, 1456 

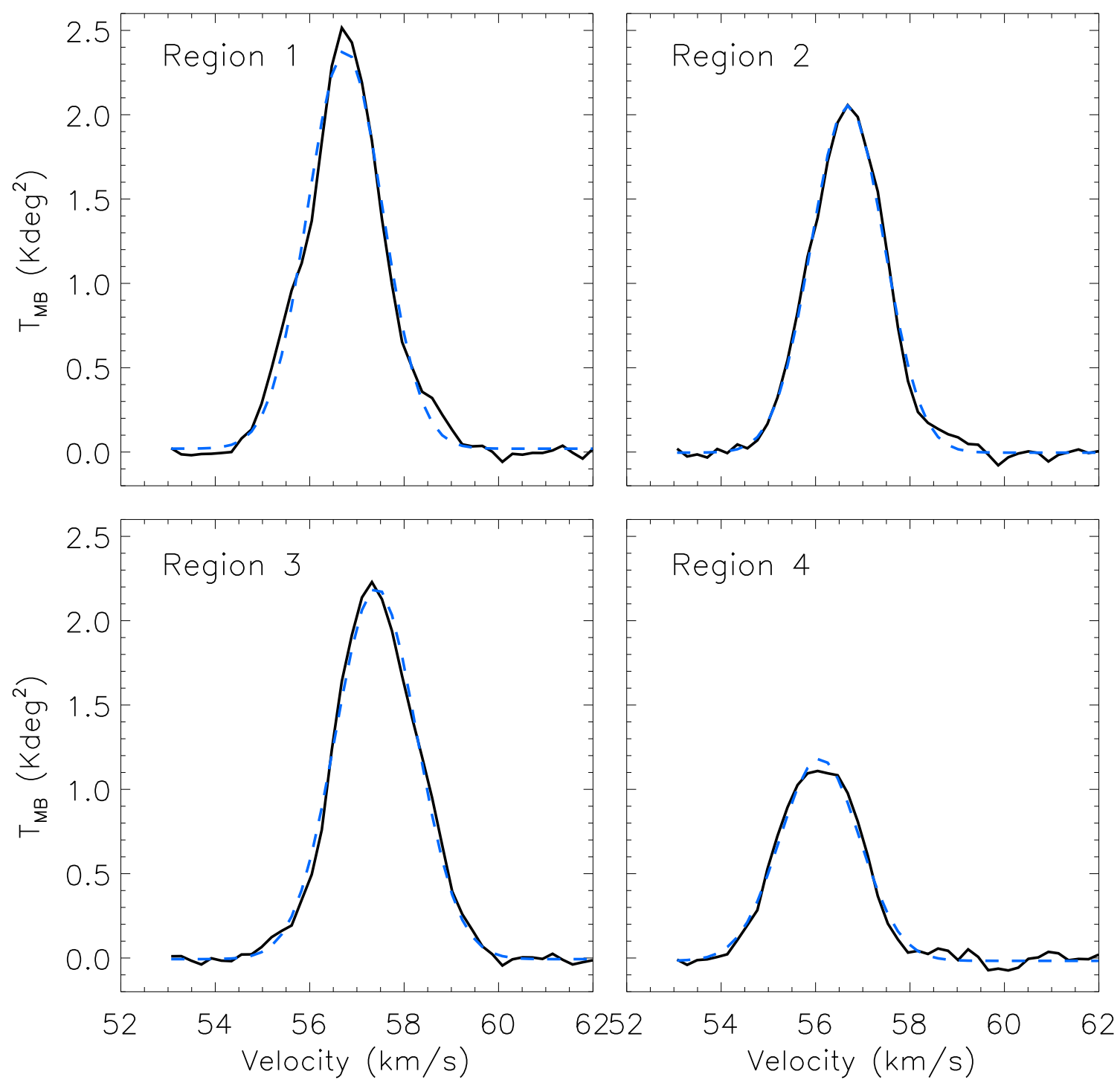

Figure 9. A mean spectrum toward each subregion (see also Figure 7 ). In each subregion, the observed mean profile is shown by a solid curve, while the dashed curve indicates the resulting Gaussian fit.

Ott, S. 2010, in Astronomical Society of the Pacic Conference Series, Vol. 434, Astronomical Data Analysis Software and Systems XIX, ed. Y. Mizumoto, K.-I. Morita, \& M. Ohishi, 139

Ostriker, E. C., Stone, J. M., \& Gammie, C. F. 2001, ApJ, 546, 980

Panagia, N. 1973, AJ, 78, 929

Panagia, N. \& Felli, M. 1975, A\&A, 39, 1

Panagia, N., \& Walmsey, C. M. 1978, A\&A, 70, 711

Paladini R., Umana G., Veneziani M., et al. 2012, ApJ, 760, 149

Paron, S., Petriella, A. \& Ortega, M. E. 2011, A\&A, 525, 132

Pavel, M. D., \& Clemens, D. P. 2012, ApJ, 760, 150

Peters, T., Mac Low, M.-M., Banerjee, R., Klessen, R. S., \& Dullemond, C. P. 2010, ApJ, 719, 831

Pilbratt, G. L., Riedinger, J. R., Passvogel, T., et al. 2010, A\&A, 518, L1

Planck Collaboration IX. 2014, A\&A, 571, A9

Planck Collaboration I. 2016a, A\&A, 594, A1

Planck Collaboration VIII. 2016b, A\&A, 594, A8

Planck Collaboration XXXV. 2016c, A\&A, 586, A138

Plaszczynski, S., Montier, L., Levrier, F., \& Tristram, M. 2014, MNRAS, 439, 4048
Poglitsch, A., Waelkens, C., Geis, N., et al. 2010, A\&A, 518L, 2 Purcell, C. R., Hoare, M. G., Cotton, W. D., et al. 2013, ApJS, 205, 1

Reich, W., Fuerst, E., Haslam, C. G. T., Steffen, P., \& Reif, K. 1984, A\&AS, 58, 197

Rebull, L. M., Guieu, S., Stauffer, J. R., et al. 2011, ApJS, 193, 25

Sánchez-Monge, Á., Kurtz, S., Palau, A., et al. 2013, ApJ, 766, 114

Skrutskie, M. F., Cutri, R. M., Stiening, R., et al. 2006, AJ, 131, 1163

Smith, L. J., Norris, R. P. F., \& Crowther, P. A. 2002, MNRAS, 337, 1309

Thompson, M. A., Urquhart, J. S., Moore, T. J. T., Morgan, L. K. 2012, MNRAS, 421, 408

Treviño-Morales, S. P., Fuente, A., Sánchez-Monge, Á., et al. 2016, A\&A, 593L, 12

Williams, J. P., de Geus, E. J., \& Blitz, L. 1994, ApJ, 428, 693 Wright, E. L., Eisenhardt, P. R. M., Mainzer, A. K., et al. 2010 , AJ, 140, 1868 

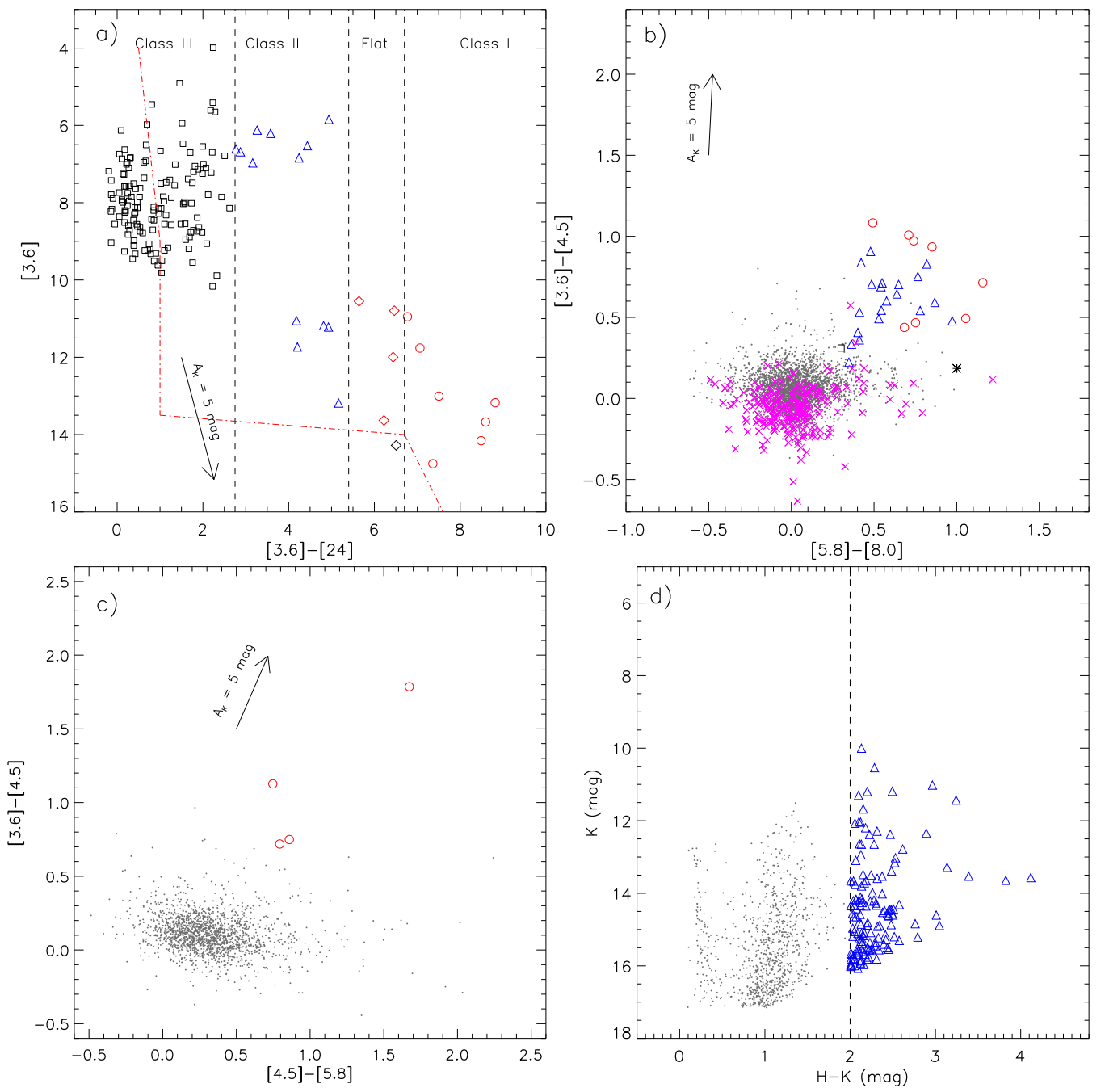

Figure 10. Identification of young stellar populations in the region around the G35.6 site. a) The panel shows a color-magnitude plot ([3.6] - [24] vs [3.6]) of sources detected in the Spitzer 3.6 and $24 \mu \mathrm{m}$ bands (see the text for more details). The plot traces YSOs belonging to different evolutionary stages (see dashed lines). The zone of YSOs against contaminated candidates (galaxies and disk-less stars) is highlighted by dotted-dashed lines (in red) (see Rebull et al. 2011 for more details). Flat-spectrum and Class III sources are marked by "ऽ" and " $\square$ " symbols, respectively. b) The panel shows a color-color plot ([3.6]-[4.5] vs. [5.8]-[8.0]) of sources detected in the Spitzer-GLIMPSE 3.6-8.0 $\mu \mathrm{m}$ bands. The PAH-emitting galaxies and the PAH-emission-contaminated apertures are marked by "*" and "X" symbols, respectively (see the text). A Class III source is highlighted by "口" symbol in the panel. c) The panel shows a color-color plot ([3.6]-[4.5] vs. [4.5]-[5.8]) of sources detected in the Spitzer-GLIMPSE 3.6-5.8 $\mu \mathrm{m}$ bands. d) The panel shows a color-magnitude plot (H-K/K) of sources selected from the GPS and $2 \mathrm{MASS}$ data. In all the panels, we show Class I (red circles) and Class II (open blue triangles) YSOs. In the last three panels, the dots in gray color show the stars with only photospheric emission. In the NIR $\mathrm{H}-\mathrm{K} / \mathrm{K}$ plot, we have plotted only 901 out of 49399 stars with photospheric emission. Due to large numbers of stars with photospheric emission, only some of these stars are randomly marked in the NIR H-K/K plot. In the first three panels (i.e., color-color diagrams), an extinction vector for $\mathrm{A}_{V}=5 \mathrm{mag}$ is drawn and is derived using the average extinction laws from Flaherty et al. (2007). 

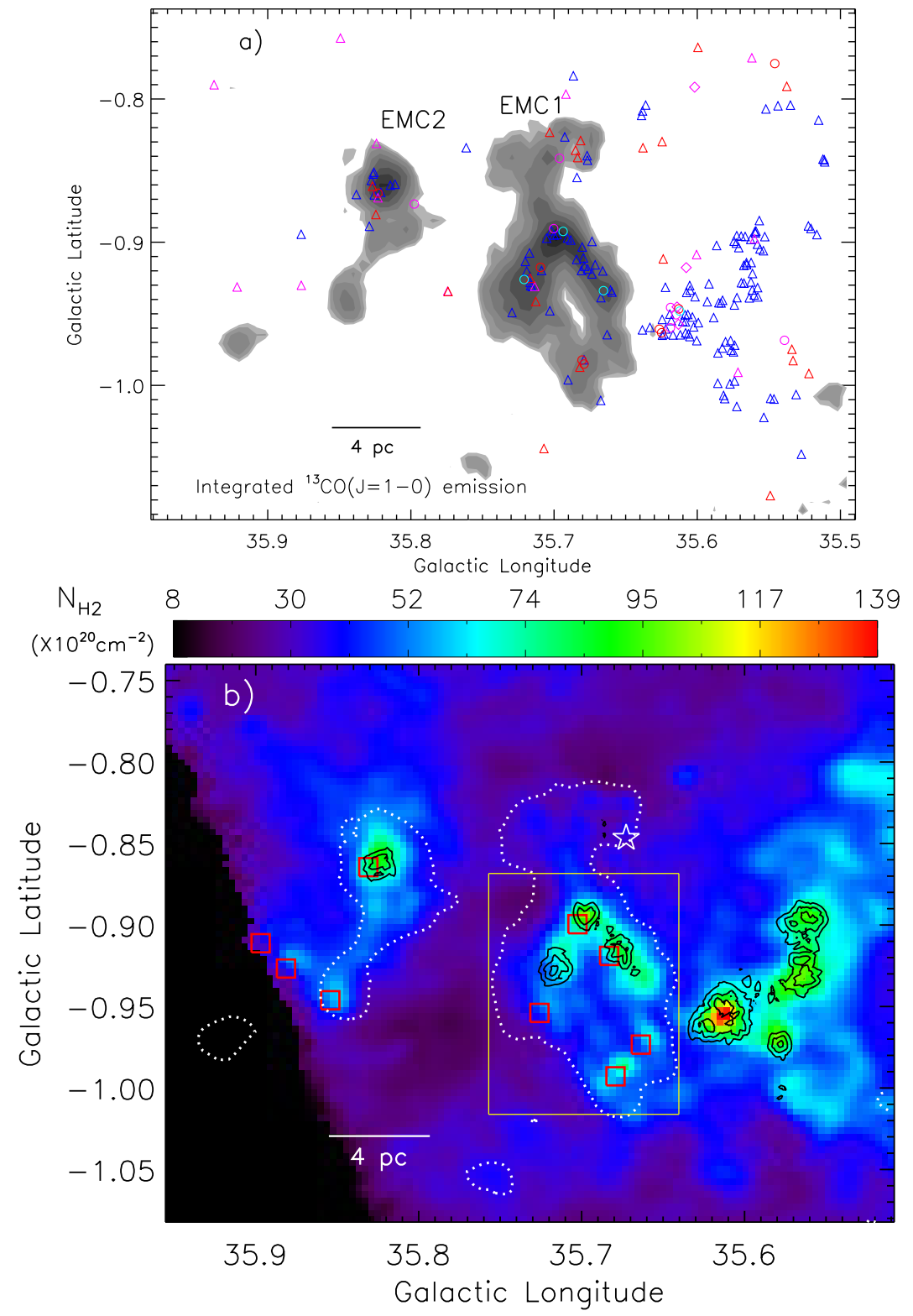

Figure 11. Spatial distribution of young stellar populations in the region around the G35.6 site. a) The selected young stellar populations (Class I (circles), Flat-spectrum (diamond), and Class II (triangles)) are shown in the integrated ${ }^{13} \mathrm{CO}$ map. The background map is similar to the one shown in Figure 5 . The YSOs identified using the Spitzer color-magnitude scheme (i.e. [3.6] - [24] vs [3.6]; see Figure 10p), four Spitzer-GLIMPSE 3.6-8.0 $\mu \mathrm{m}$ bands (see Figure 10 ), three Spitzer-GLIMPSE 4.5-8.0 $\mu \mathrm{m}$ bands (see Figure 10 ), and NIR color-magnitude scheme (i.e. H-K/K; see Figure 10 $\mathrm{d}$ ) are shown by magenta, red, cyan, and blue color symbols, respectively. The molecular map helps us to infer the physical association of YSOs with the MCG35.6. b) Herschel column density map is overlaid with surface density contours (in black) of all the identified YSOs and also Herschel clumps. The surface density contours are drawn at 3,5 , and $10 \mathrm{YSOs} / \mathrm{pc}^{2}$, increasing from the outer to the inner regions. The background map is similar to the one shown in Figure 6p. The Herschel clumps are also highlighted by squares. The solid yellow box encompasses the area shown in Figures 12 and 12 


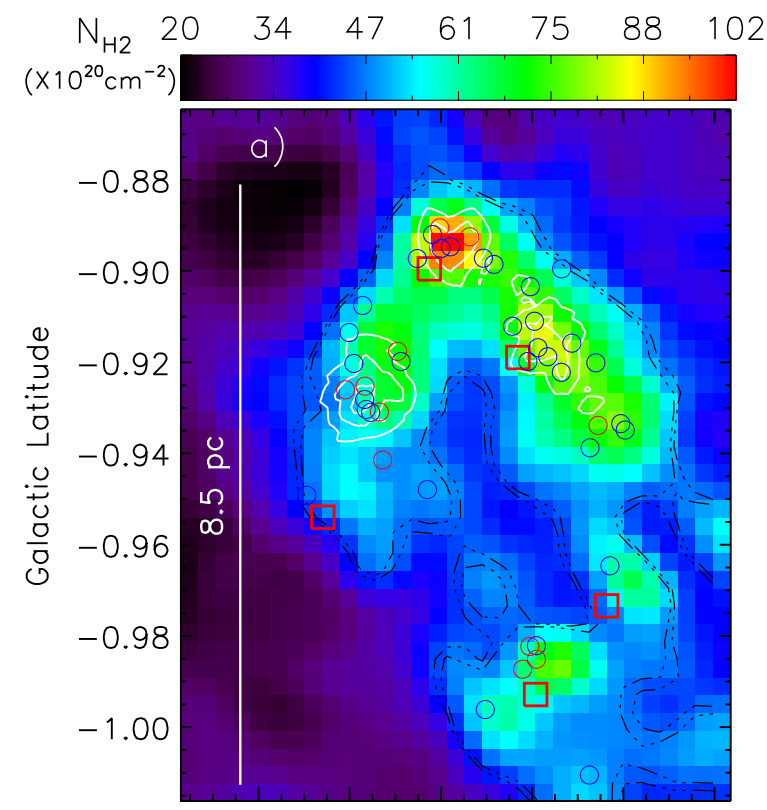

35.7435.7235.7035.6835.6635.64 Galactic Longitude

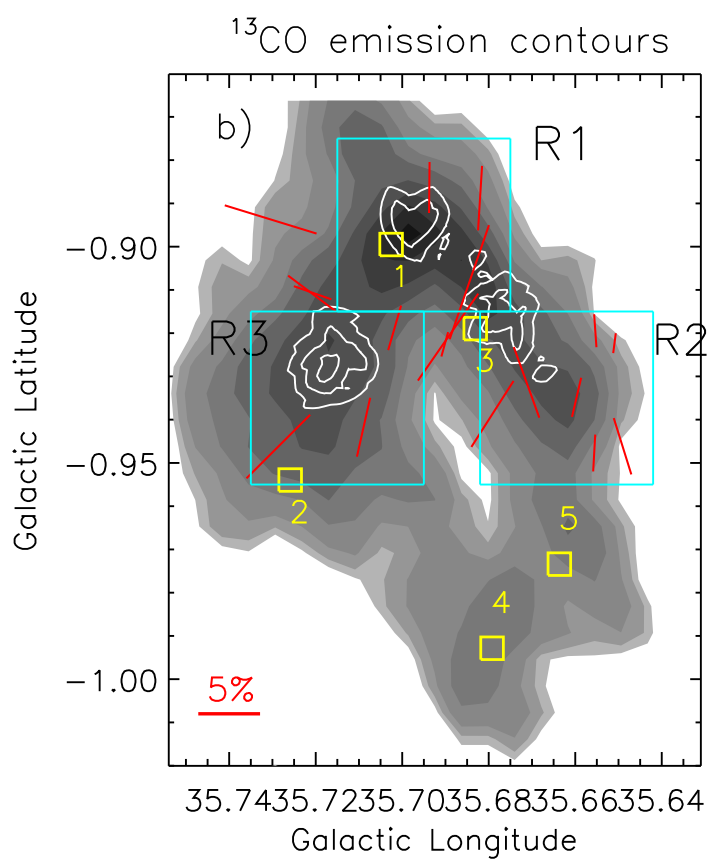

Figure 12. Zoomed-in view of the ring-like feature. a) Herschel column density map is superimposed with surface density contours (in white) of all the identified YSOs, Herschel clumps and selected YSOs. b) Overlay of GPIPS H-band polarization vectors (in red) of high quality background stars on the integrated ${ }^{13} \mathrm{CO}$ emission map (see Table 3 . The map is also superimposed with surface density contours (in white) of all the identified YSOs and the Herschel clumps. A reference vector of $5 \%$ is highlighted in the lower left corner of the map (see also Figure 8 ). In both the panels, the background maps and other marked symbols are similar to the one shown in Figures $8 \mathrm{a}$ and $8 \mathrm{p}$. 
Table 3

2MASS photometric and GPIPS H-band polarimetric parameters of 17 stars seen toward the ring-like feature. Table lists 2MASS magnitudes (J-, H-, and K- bands), linear polarization $(\mathrm{P})$, uncertainty in $\mathrm{P}(\mathrm{sP})$, Galactic position angle (GPA), and uncertainty in position angle (sPA) (see Figure 8p).

\begin{tabular}{lcccccccc}
\hline$l$ & $\begin{array}{c}\text { J-band } \\
(\mathrm{deg})\end{array}$ & $\begin{array}{c}\text { H-band } \\
(\mathrm{deg})\end{array}$ & $\begin{array}{c}\text { K-band } \\
(\mathrm{mag})\end{array}$ & $\begin{array}{c}\mathrm{P} \\
(\mathrm{mag})\end{array}$ & $\begin{array}{c}\text { sP } \\
(\%)\end{array}$ & $\begin{array}{c}\text { GPA } \\
(\mathrm{deg})\end{array}$ & $\begin{array}{c}\text { sPA } \\
(\mathrm{deg})\end{array}$ \\
\hline \hline 35.682 & -0.888 & 15.54 & 11.97 & 10.22 & 5.23 & 1.77 & 176.41 & 9.69 \\
35.655 & -0.919 & 12.01 & 9.61 & 8.47 & 2.67 & 0.33 & 3.94 & 3.57 \\
35.650 & -0.922 & 11.71 & 10.50 & 10.02 & 1.62 & 0.28 & 172.62 & 5.07 \\
35.684 & -0.908 & 16.63 & 12.98 & 11.27 & 9.76 & 2.09 & 160.99 & 6.14 \\
35.659 & -0.934 & 16.84 & 12.82 & 10.53 & 3.24 & 1.31 & 166.80 & 11.6 \\
35.689 & -0.920 & 15.61 & 12.86 & 11.59 & 8.56 & 2.46 & 145.21 & 8.24 \\
35.671 & -0.931 & 15.37 & 12.67 & 11.43 & 6.07 & 1.72 & 19.81 & 8.13 \\
35.690 & -0.922 & 11.36 & 8.81 & 7.45 & 2.04 & 0.57 & 164.50 & 8.05 \\
35.649 & -0.946 & 15.81 & 12.03 & 10.31 & 4.77 & 1.06 & 17.35 & 6.37 \\
35.655 & -0.947 & 12.56 & 10.12 & 9.00 & 2.98 & 0.23 & 176.77 & 2.23 \\
35.679 & -0.938 & 13.70 & 12.32 & 11.55 & 6.33 & 1.45 & 147.44 & 6.59 \\
35.693 & -0.886 & 15.55 & 11.76 & 9.79 & 4.12 & 1.58 & 179.38 & 11.01 \\
35.730 & -0.893 & 14.50 & 12.78 & 11.92 & 7.77 & 2.61 & 72.90 & 9.63 \\
35.701 & -0.918 & 13.60 & 11.85 & 11.15 & 3.75 & 1.49 & 163.90 & 11.43 \\
35.720 & -0.910 & 14.65 & 11.94 & 10.66 & 3.21 & 1.45 & 70.58 & 12.93 \\
35.708 & -0.941 & 14.00 & 11.42 & 10.23 & 4.88 & 1.17 & 167.34 & 6.90 \\
35.728 & -0.946 & 15.20 & 12.57 & 11.35 & 7.27 & 3.28 & 134.94 & 12.91 \\
\hline
\end{tabular}

Table 4

Physical properties of four subregions selected in EMC1 (see Figure $7 \mathrm{k}$ ). Table lists subregions, positions $(l$ and $b)$, PA dispersion $(\alpha)$, velocity dispersion $\left(\sigma_{v}\right)$, volume density $\left(n_{H_{2}}\right),\left\langle B_{\text {pos }}\right\rangle$, magnetic pressure $\left(P_{\text {mag }}=B_{\text {pos }}^{2} / 8 \pi\right)$, and normalized mass-to-flux ratio $\left(\overline{M / \Phi_{B}}\right)$.

\begin{tabular}{lcccccccc}
\hline subregion & $\begin{array}{c}l \\
(\text { degree })\end{array}$ & $\begin{array}{c}b \\
\text { (degree) })\end{array}$ & $\begin{array}{c}\alpha \\
(\text { degree })\end{array}$ & $\begin{array}{c}\sigma_{v} \\
\left(\mathrm{~km} \mathrm{~s}^{-1}\right)\end{array}$ & $\begin{array}{c}n_{\mathrm{H}_{2}} \\
\left(\mathrm{~cm}^{-3}\right)\end{array}$ & $\begin{array}{c}<B_{\text {pos }}> \\
(\mu \mathrm{G})\end{array}$ & $\begin{array}{c}P_{\text {mag }} \\
\left(\times 10^{-11} \text { dynes cm }^{-2}\right)\end{array}$ & $\overline{M / \Phi_{B}}$ \\
\hline \hline 1 & 35.695 & -0.895 & 9.9 & 0.79 & 469 & 21.5 & 1.8 & 1.5 \\
2 & 35.665 & -0.935 & 18.1 & 0.73 & 465 & 11.0 & 0.48 & 3.0 \\
3 & 35.715 & -0.935 & 17.8 & 0.78 & 407 & 11.0 & 0.48 & 2.6 \\
4 & 35.725 & -0.845 & 7.0 & 0.68 & 275 & 20.0 & 1.6 & 0.4 \\
\hline
\end{tabular}


Table 5

NIR and MIR photometric magnitudes are listed for 59 YSOs (8 Class I and 51 Class II) distributed toward EMC1. These YSOs are identified using the color-magnitude and color-color schemes. The Spitzer color-magnitude scheme ([3.6] - [24] vs [3.6]), four GLIMPSE bands color-color scheme ([3.6] - [4.5] vs. [5.8]-[8.0]), three GLIMPSE bands color-color scheme ([3.6] - [4.5] vs. [4.5] - [5.8]), and NIR color-magnitude scheme $(\mathrm{H}-\mathrm{K} / \mathrm{K})$ are referred to as $\mathrm{sh} 1, \operatorname{sh} 2, \operatorname{sh} 3$, and $\operatorname{sh} 4$, respectively.

\begin{tabular}{|c|c|c|c|c|c|c|c|c|c|c|c|}
\hline ID & $\begin{array}{c}\text { YSO } \\
\text { (stage,scheme) }\end{array}$ & $\begin{array}{c}l \\
\text { (degree) }\end{array}$ & $\begin{array}{c}b \\
\text { (degree) }\end{array}$ & $\begin{array}{c}\mathrm{J} \\
(\mathrm{mag})\end{array}$ & $\begin{array}{c}\mathrm{H} \\
(\mathrm{mag})\end{array}$ & $\begin{array}{c}\mathrm{K} \\
(\mathrm{mag}) \\
\end{array}$ & $\begin{array}{c}3.6 \mu \mathrm{m} \\
(\mathrm{mag})\end{array}$ & $\begin{array}{c}4.5 \mu \mathrm{m} \\
(\mathrm{mag})\end{array}$ & $\begin{array}{c}5.8 \mu \mathrm{m} \\
(\mathrm{mag})\end{array}$ & $\begin{array}{c}8.0 \mu \mathrm{m} \\
(\mathrm{mag})\end{array}$ & $\begin{array}{l}24 \mu \mathrm{m} \\
(\mathrm{mag})\end{array}$ \\
\hline 1 & $\overline{\mathrm{I} \text { I,sh1 }}$ & ב35.696 & "-0.841 & $\overline{-1-}$ & - & - - & $\bar{~} 13.18$ & "11.30 & 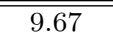 & 8.56 & 4.36 \\
\hline 2 & I,sh1 & 35.700 & -0.891 & - & - & 13.45 & 10.95 & 9.76 & 8.86 & 8.32 & 4.18 \\
\hline 3 & II,sh1 & 35.713 & -0.931 & - & - & 13.52 & 11.18 & 10.30 & 9.74 & 9.23 & 6.37 \\
\hline 4 & I,sh2 & 35.709 & -0.918 & - & - & - & 13.58 & 13.15 & 11.98 & 11.30 & - \\
\hline 5 & I,sh2 & 35.680 & -0.982 & - & 11.46 & 9.54 & 7.42 & 6.71 & 5.99 & 4.83 & - \\
\hline 6 & $\mathrm{I}, \mathrm{sh} 2$ & 35.679 & -0.985 & - & - & 14.58 & 11.91 & 10.94 & 10.26 & 9.52 & - \\
\hline 7 & II,sh2 & 35.681 & -0.829 & 15.83 & 13.62 & 12.49 & 11.28 & 10.69 & 10.05 & 9.18 & - \\
\hline 8 & II,sh2 & 35.684 & -0.841 & - & - & - & 11.55 & 11.05 & 10.49 & 9.96 & - \\
\hline 9 & II,sh2 & 35.685 & -0.836 & - & 15.05 & 13.74 & 12.10 & 11.56 & 11.06 & 10.51 & - \\
\hline 10 & II,sh2 & 35.703 & -0.823 & 16.51 & 14.15 & 12.97 & 12.08 & 11.60 & 11.26 & 10.29 & - \\
\hline 11 & II,sh2 & 35.682 & -0.987 & - & 14.68 & 13.58 & 11.71 & 11.03 & 10.65 & 10.11 & - \\
\hline 12 & II,sh2 & 35.717 & -0.925 & 15.78 & 13.61 & 12.11 & 10.11 & 9.57 & 8.99 & 8.21 & - \\
\hline 13 & II, $\operatorname{sh} 2$ & 35.713 & -0.941 & 15.71 & 14.11 & 13.15 & 11.80 & 11.20 & 10.72 & 10.14 & - \\
\hline 14 & II,sh2 & 35.638 & -0.834 & - & - & - & 13.36 & 12.53 & 12.15 & 11.33 & - \\
\hline 15 & I,sh3 & 35.666 & -0.934 & - & - & - & 14.95 & 13.17 & 11.49 & 11.34 & - \\
\hline 16 & $\mathrm{I}, \mathrm{sh} 3$ & 35.694 & -0.892 & - & - & 14.27 & 12.43 & 11.68 & 10.82 & - & - \\
\hline 17 & $\mathrm{I}, \mathrm{sh} 3$ & 35.721 & -0.926 & - & 14.99 & 13.38 & 10.98 & 9.86 & 9.11 & 8.36 & - \\
\hline 18 & II, $\operatorname{sh} 4$ & 35.661 & -0.933 & 17.91 & 14.13 & 12.07 & 10.73 & 10.59 & 10.27 & 10.45 & - \\
\hline 19 & II, $\operatorname{sh} 4$ & 35.671 & -0.916 & 18.62 & 14.78 & 12.65 & 11.33 & 11.18 & 10.92 & - & - \\
\hline 20 & II,sh4 & 35.703 & -0.948 & 20.39 & 17.77 & 15.56 & 14.38 & 14.13 & - & - & - \\
\hline 21 & II, $\operatorname{sh} 4$ & 35.677 & -0.919 & - & 17.93 & 14.89 & 12.77 & 12.51 & - & - & - \\
\hline 22 & II, $\operatorname{sh} 4$ & 35.639 & -0.811 & 18.51 & 14.60 & 12.38 & 10.91 & 10.74 & 10.45 & 10.47 & - \\
\hline 23 & II,sh4 & 35.638 & -0.961 & - & 16.99 & 14.96 & 14.03 & 13.40 & - & - & - \\
\hline 24 & II, $\operatorname{sh} 4$ & 35.633 & -0.960 & - & 15.24 & 12.34 & 10.82 & 10.52 & 10.08 & 10.12 & - \\
\hline 25 & II, $\operatorname{sh} 4$ & 35.666 & -0.920 & - & 16.39 & 14.34 & 13.04 & 12.91 & - & - & - \\
\hline 26 & II,sh4 & 35.684 & -0.912 & - & 17.74 & 15.45 & 13.43 & 12.91 & 12.22 & - & - \\
\hline 27 & II, $\operatorname{sh} 4$ & 35.667 & -0.939 & - & 16.23 & 14.11 & 12.69 & 12.62 & - & - & - \\
\hline 28 & II, $\operatorname{sh} 4$ & 35.679 & -0.917 & - & 17.96 & 15.95 & 13.45 & 13.36 & - & - & - \\
\hline 29 & II, $\operatorname{sh} 4$ & 35.679 & -0.911 & - & 16.92 & 13.53 & 11.18 & 10.87 & 10.41 & 11.05 & - \\
\hline 30 & II,sh4 & 35.681 & -0.920 & - & 17.39 & 15.26 & 13.62 & 13.26 & - & - & - \\
\hline 31 & II, $\operatorname{sh} 4$ & 35.680 & -0.903 & - & 14.68 & 11.43 & 9.34 & 9.07 & 8.55 & 8.59 & - \\
\hline 32 & II, $\operatorname{sh} 4$ & 35.663 & -0.965 & - & 17.71 & 15.20 & 13.76 & 13.62 & - & - & - \\
\hline 33 & II,sh4 & 35.674 & -0.922 & - & 17.06 & 14.57 & 12.99 & 12.67 & 11.66 & - & - \\
\hline 34 & II,sh4 & 35.674 & -0.900 & - & 18.05 & 16.00 & 14.46 & 14.03 & - & - & - \\
\hline 35 & II, $\operatorname{sh} 4$ & 35.720 & -0.913 & - & 17.32 & 15.16 & 13.85 & 13.53 & - & - & - \\
\hline 36 & II,sh4 & 35.700 & -0.895 & - & 18.02 & 16.02 & - & - & - & - & - \\
\hline 37 & II, $\operatorname{sh} 4$ & 35.688 & -0.899 & - & 17.99 & 15.86 & 14.37 & 13.99 & - & - & - \\
\hline 38 & II,sh4 & 35.636 & -0.804 & - & 15.40 & 12.78 & 11.13 & 10.81 & 10.42 & 10.30 & - \\
\hline 39 & II,sh4 & 35.690 & -0.996 & - & 16.98 & 14.85 & - & - & - & - & - \\
\hline 40 & II,sh4 & 35.667 & -1.011 & - & 17.32 & 15.08 & 13.69 & 13.53 & - & - & - \\
\hline 41 & II, $\operatorname{sh} 4$ & 35.761 & -0.834 & 17.80 & 16.89 & 14.78 & - & - & - & - & - \\
\hline 42 & II,sh4 & 35.677 & -0.843 & 19.74 & 16.46 & 14.34 & 12.22 & 11.53 & 10.91 & - & - \\
\hline 43 & II,sh4 & 35.717 & -0.908 & 17.33 & 13.40 & 11.30 & 9.97 & 9.87 & 9.40 & 9.29 & - \\
\hline 44 & II,sh4 & 35.705 & -0.897 & 19.30 & 14.60 & 12.29 & 10.82 & 10.70 & 10.22 & 10.19 & - \\
\hline 45 & II,sh4 & 35.684 & -0.855 & 18.89 & 14.85 & 12.38 & 10.91 & 10.58 & 10.25 & 10.49 & - \\
\hline 46 & II,sh4 & 35.717 & -0.928 & 19.24 & 15.67 & 13.66 & 12.52 & 12.12 & 11.78 & - & - \\
\hline 47 & II,sh4 & 35.715 & -0.931 & 19.48 & 15.06 & 12.94 & 11.61 & 11.33 & 10.96 & 10.83 & - \\
\hline 48 & II,sh4 & 35.719 & -0.920 & - & 17.89 & 15.56 & 14.06 & 13.85 & - & - & - \\
\hline 49 & II,sh4 & 35.677 & -0.840 & - & 17.85 & 15.68 & 13.66 & 13.28 & - & - & - \\
\hline 50 & II,sh4 & 35.702 & -0.892 & - & 17.68 & 15.54 & 14.16 & 13.93 & - & - & - \\
\hline 51 & II,sh4 & 35.698 & -0.895 & - & 17.97 & 15.85 & - & - & - & - & - \\
\hline 52 & II,sh4 & 35.709 & -0.920 & - & 17.35 & 14.87 & 13.48 & 12.85 & - & - & - \\
\hline 53 & II,sh4 & 35.691 & -0.897 & - & 17.87 & 15.30 & 13.65 & 13.12 & - & - & - \\
\hline 54 & II,sh4 & 35.638 & -0.808 & - & 17.60 & 14.84 & 12.94 & 12.42 & 12.11 & - & - \\
\hline 55 & II,sh4 & 35.693 & -0.827 & - & 17.83 & 15.80 & 13.35 & 13.37 & - & - & - \\
\hline 56 & II,sh4 & 35.729 & -0.949 & - & 17.02 & 14.81 & 13.43 & 13.19 & - & - & - \\
\hline 57 & II,sh4 & 35.716 & -0.930 & 一 & 16.99 & 14.56 & 13.01 & 12.65 & - & - & - \\
\hline 58 & II,sh4 & 35.679 & -0.982 & 15.36 & 14.16 & 12.04 & 10.25 & 9.92 & 9.25 & 8.52 & - \\
\hline 59 & II,sh4 & 35.660 & -0.935 & 16.84 & 12.82 & 10.54 & 8.69 & 8.50 & 8.01 & 7.90 & 6.04 \\
\hline
\end{tabular}

